\title{
Desialylation is a mechanism of Fc-independent platelet clearance and a therapeutic target in immune thrombocytopenia
}

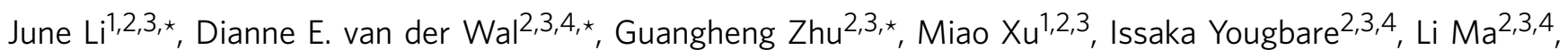
Brian Vadasz ${ }^{1,2,3}$, Naadiya Carrim ${ }^{2,3}$, Renata Grozovsky ${ }^{5}$, Min Ruan ${ }^{6}$, Lingyan Zhu6 ${ }^{6}$, Qingshu Zeng ${ }^{6}$, Lili Tao ${ }^{6}$, Zhi-min Zhai ${ }^{6}$, Jun Peng ${ }^{7}$, Ming Hou', Valery Leytin ${ }^{1,2,3}$, John Freedman1,2,3,8, Karin M. Hoffmeister ${ }^{5}$ \& Heyu $\mathrm{Ni}^{1}, 2,3,4,8,9$

Immune thrombocytopenia (ITP) is a common bleeding disorder caused primarily by autoantibodies against platelet GPIIbllla and/or the GPIb complex. Current theory suggests that antibody-mediated platelet destruction occurs in the spleen, via macrophages through $\mathrm{Fc}-\mathrm{Fc} \gamma \mathrm{R}$ interactions. However, we and others have demonstrated that anti-GPIb $\alpha$ (but not GPIIbllla)-mediated ITP is often refractory to therapies targeting Fc $\gamma R$ pathways. Here, we generate mouse anti-mouse monoclonal antibodies (mAbs) that recognize GPIb $\alpha$ and GPIIbllla of different species. Utilizing these unique mAbs and human ITP plasma, we find that anti-GPIb $\alpha$, but not anti-GPIIbllla antibodies, induces Fc-independent platelet activation, sialidase neuraminidase- 1 translocation and desialylation. This leads to platelet clearance in the liver via hepatocyte Ashwell-Morell receptors, which is fundamentally different from the classical Fc-Fc $\gamma R$-dependent macrophage phagocytosis. Importantly, sialidase inhibitors ameliorate anti-GPIb $\alpha$-mediated thrombocytopenia in mice. These findings shed light on Fc-independent cytopenias, designating desialylation as a potential diagnostic biomarker and therapeutic target in the treatment of refractory ITP.

\footnotetext{
${ }^{1}$ Department of Laboratory Medicine and Pathobiology, University of Toronto, Toronto, Ontario, Canada M5S 1A8. ${ }^{2}$ Toronto Platelet Immunobiology Group, Toronto, Ontario, Canada M5B 1W8. ${ }^{3}$ Department of Laboratory Medicine, Keenan Research Centre for Biomedical Science of St. Michael's Hospital, Toronto, Ontario, Canada M5B 1W8. ${ }^{4}$ Canadian Blood Services, Ottawa, Ontario, Canada K1G 4J5. ${ }^{5}$ Translational Medicine Division, Brigham and Women's Hospital, Harvard Medical School, Boston, Massachusetts 02115, USA. ${ }^{6}$ Department of Hematology, Anhui Medical University, Hefei 230032, China. ${ }^{7}$ Department of Hematology, Qilu Hospital, Shandong University, Jinan 250012, China. ${ }^{8}$ Department of Medicine, University of Toronto, Ontario, Canada M5S $1 A 8$.

${ }^{9}$ Department of Physiology, University of Toronto, Ontario, Canada M5S 1A8. * These authors contributed equally to this work. Correspondence and requests for materials should be addressed to H.N. (email: nih@smh.ca).
} 
mmune thrombocytopenia (ITP) is a common bleeding disorder characterized by increased destruction of autologous platelets $^{1-3}$. Low platelet counts increase the risk for bleeding, which leads to severe intracranial haemorrhage in $\sim 5 \%$ of patients ${ }^{1-3}$. ITP patients live with the risk of fatal bleeding and many undergo long-term therapeutic regimens to manage platelet counts, and suffer a marked decrease in quality of life ${ }^{4}$. First-line treatments include immunosuppressive and immunomodulatory agents (that is, corticosteroids, intravenous immunoglobulin $G$ (IVIG) and anti-RhD therapy). Splenectomy has to be considered for patients with a persistent lack of response to treatment ${ }^{5}$. However, it is estimated that $15-25 \%$ of patients are inexplicably refractory to first-line therapies and even splenectomy ${ }^{6}$. To date, there is no reliable measurement in the clinical setting to predict the success or failure of any ITP treatment ${ }^{5,7}$.

Autoantibodies targeting platelet surface glycoprotein(s) (GP) have been demonstrated to be the major factors responsible for platelet clearance $2,8,9$. Approximately $70-80 \%$ of patients have autoantibodies against GPIIbIIIa (integrin $\alpha \mathrm{IIb} \beta 3$ ), $20-40 \%$ against the GPIb complex and some patients have autoantibodies against both or other GPs ${ }^{11-13}$. Platelet destruction following autoantibody binding has generally been considered to occur in the spleen, through binding of the Fc portion of immunoglobulins on the platelet surface to Fc $\gamma$ RIIa and Fc $\gamma$ RIIIa on tissue macrophages of the reticuloendothelial system ${ }^{2}$. Accordingly, first-line therapies, such as IVIG and anti-Rh(D), target these Fc- and Fc $\gamma \mathrm{R}$-dependent mechanisms to restore platelet numbers ${ }^{10}$. Unexpectedly, we and others have identified a novel mechanism of Fc-independent thrombocytopenia, in which antibodies against GPIb $\alpha$, but not those against GPIIbIIIa, can induce thrombocytopenia via their $\mathrm{F}(\mathrm{ab})_{2}$ ( $\mathrm{Fc}$ independent) and in $F c \gamma R^{-/-}$mice ${ }^{11,12}$. We further reported that most anti-GPIb $\alpha$ antibody-mediated thrombocytopenia is resistant to IVIG treatment ${ }^{12}$. This is consistent with subsequent reports in humans, including our recent large patient cohort study ${ }^{13-15}$. In addition, our retrospective studies suggest that ITP patients with anti-GPIb $\alpha$ antibodies are also more likely to be refractory to steroid treatments ${ }^{16}$. These data indicate that anti-GPIb $\alpha$ antibodies are able to uniquely induce platelet clearance in an Fc-independent manner in murine models, which may also be true in human ITP. However, the nature of this novel Fc-independent mechanism of platelet clearance is unknown.

GPIIbIIIa and the GPIb complex are structurally and functionally distinct platelet receptors. Although different outside-in signalling pathways have been observed between these two receptors following ligand stimulation ${ }^{17,18}$, the downstream effects of autoantibody binding have not been adequately studied. Thus, possible differences in pathogenesis and therapy between anti-GPIIbIIIa- and anti-GPIb-mediated ITP remain to be elucidated.

As the second-most abundant platelet surface receptor, GPIb $\alpha$ is the largest subunit and possesses all known extracellular ligandbinding sites of the GPIb complex (that is, GPIb-IX-V). Binding of GPIb $\alpha$ to the von Willebrand factor initiates GPIb $\alpha$ outside-in signalling, which can subsequently activate GPIIbIIIa leading to platelet aggregation ${ }^{17,19}$. GPIb $\alpha$ is also the most heavily glycosylated platelet surface protein with $\sim 60 \%$ carbohydrate by weight ${ }^{20}$. It contains both $N$ - and $O$-linked carbohydrate chains capped with sialic acid, contributing to $64 \%$ of the total sialic acid content of the platelet ${ }^{21}$. Deglycosylation of GPIb $\alpha$ was reported to result in hepatic clearance of cold-stored platelets following transfusion ${ }^{22-24}$. This suggests that glycan modification of GPIb $\alpha$ may be sufficient to induce platelet clearance, likely via an Fc-independent pathway. We previously found that antisera against GPIb $\alpha$ can induce platelet activation ${ }^{25}$; however, other downstream platelet responses following antibody binding have not been adequately studied. Whether antibody binding may also lead to deglycosylation and contribute to platelet clearance, and whether this may account for the differences in pathogenesis and response to therapy in ITP has not been investigated.

In this study, by utilizing unique anti-GPIb $\alpha$ and antiGPIIbIIIa monoclonal antibodies (mAbs) as well as human ITP plasma samples, we show that significant platelet activation and desialylation occurs predominantly in the presence of anti-GPIb $\alpha$, but not anti-GPIIbIIIa antibodies in vitro and in vivo. Furthermore, anti-GPIb $\alpha$-mediated desialylation of platelets led to Fc $\gamma$ R-independent clearance via hepatocytes. Most importantly, the use of sialidase inhibitors mitigated thrombocytopenia in a murine model of anti-GPIb $\alpha$-mediated ITP. Thus, we identify a mechanism of platelet clearance previously undescribed. In addition, we introduce a new diagnostic method to detect platelet desialylation in ITP and establish sialidase inhibitors as a potential therapeutic for refractory ITP.

\section{Results}

Generation of novel anti-GPIb $\alpha$ and anti-GPIIbIIIa antibodies. To examine the effects of anti-GPIb $\alpha$ and anti-GPIIbIIIa antibodies on platelet function and clearance, we utilized genedeficient $\left({ }^{-/}\right)$mice to generate a panel of mouse anti-mouseGPIb $\alpha$ and -GPIIbIIIa mAbs, which possess cross-reactivity against other species, including tested human, rat and/or pig antigens (Table 1). These are the first syngeneic anti-GPIb $\alpha$ antibodies utilized in the study of ITP, thus circumventing xenogeneic antibody complications. To our knowledge, this is also the first report that anti-GPIb $\alpha$ antibodies can cross-react with GPIb $\alpha$ from different species. Further characterization of these antibodies revealed that some of these mAbs affect ristocetin/botrocetin- or ADP/thrombin-induced platelet aggregation, which are mediated by GPIb $\alpha^{26}$ or GPIIbIIIa, respectively (Table 1$)^{27-29}$. Injection of these antibodies into mice induced significant platelet decrease (thrombocytopenia) with no significant effects on red or white blood cell counts (Supplementary Table 1). Thus, these mAbs may serve useful in the study of different animal models of human diseases, including thrombosis and hemostasis, as well as other immune-mediated thrombocytopenias.

Anti-GPIb $\alpha$ antibodies induce significant platelet activation. It has been previously reported that antibodies against GPIb $\alpha$ may induce platelet activation $25,30,31$. Therefore, we investigated whether our newly developed anti-GPIb $\alpha$ mAbs may also affect platelet function. We incubated murine and human platelets with various anti-GPIb $\alpha$ or anti-GPIIbIIIa mAbs of different immunoglobulin (Ig)G subclasses (Table 1). Three anti-GPIb $\alpha$ mAbs (NIT A, NIT B and NIT F) were employed in our human platelet studies as they were, to the best of our knowledge, the first and only currently available mAbs that are cross-reactive to human GPIb $\alpha$.

Platelet granule secretion (surface expression of P-selectin) was significantly increased in the presence of all anti-GPIb $\alpha$ mAbs tested in both mouse and human platelets (Fig. 1a,e). To circumvent biases stemming from the specific binding epitopes of individual antibody clones, we also tested polyclonal sera against GPIb $\alpha$ and GPIIbIIIa (generated in knockout mice immunized with wild-type (WT) platelets ${ }^{25}$ ). Similarly, we found anti-GPIb $\alpha$, but not anti-GPIIbIIIa, sera induced platelet P-selectin expression dose dependently (Fig. 1b). Consistent with P-selectin expression, we detected significantly increased platelet GPIIbIIIa activation following anti-GPIb $\alpha$ treatment, as measured by JON/A (Fig. 1c,d) and PAC-1 binding (Fig. 1f). JON/A and PAC-1 are specific mAbs against murine and human 
GPIIbIIIa, respectively, that bind exclusively to the cationdependent high-affinity conformation of GPIIbIIIa ${ }^{32,33}$. Anti-GPIb $\alpha$-induced platelet aggregation was also visualized by light microscopy (Supplementary Fig. 1). None of the antiGPIIbIIIa antibodies tested induced detectable increases in murine platelet P-selectin expression (Fig. 1a,b). However, some anti-GPIIbIIIa antibodies, in particular $\mathrm{mAb}$ clone 9D2, did induce significant P-selectin expression on human platelets of certain (3 of the 10) healthy donors (Fig. 1e). We suspected this may have been due to immune-complex binding of Fc $\gamma$ RIIa expressed exclusively on human but not murine platelets ${ }^{34}$, which has been previously shown to cause platelet granule release independent of additional agonists ${ }^{35}$. To test this possibility, we utilized the Fc $\gamma$ RIIa/III blocker IV.3, and found that it completely attenuated 9D2-mediated P-selectin expression. Interestingly, IV.3 did not have any significant effect on any anti-GPIb $\alpha$ mAb-mediated human platelet activation (Fig. $1 \mathrm{~g}$ ).

Finally, utilizing plasma samples obtained from a cohort of ITP patients, we found anti-GPIb $\alpha$ ITP plasma induced significant P-selectin expression, while anti-GPIIbIIIa ITP plasma induced only moderate P-selectin expression (Fig. 1h). These experiments demonstrate that antibodies against GPIb $\alpha$ induced higher platelet activation, while anti-GPIIbIIIa antibodies may induce platelet activation in some human platelets through an Fc $\gamma$ RIIa-dependent mechanism (Fig. 1g). These differences between anti-GPIb $\alpha$ and anti-GPIIbIIIa antibody-induced platelet activation may serve as the basis for the mechanistic differences between anti-GPIIbIIIa Fc-dependent and anti-GPIb $\alpha$ Fc-independent platelet clearance.

Anti-GPIb $\alpha$ antibodies induce platelet desialylation. Antibodymediated platelet desialylation has not been previously investigated. To test whether anti-GPIb $\alpha$ antibodies also induce platelet desialylation, we quantified the binding of fluorescein-conjugated Ricinus communis agglutinin I (RCA-1) lectins, which specifically target exposed galactose residues following GP desialylation ${ }^{23,36}$. We found that all anti-GPIb $\alpha$ mAb-treated platelets (both murine and human) exhibited significant desialylation (Fig. 2a,e). This desialylation was dose dependent, with increasing concentrations of both our mAb (Fig. 2b,f) and GPIb $\alpha$ antisera (Fig. 2c). Platelet glycosylation changes were further characterized with additional lectins including peanut agglutinin (PNA), Sambucus Nigra Lectin and Maackia Amurensis Lectin II.
Although only significantly increased binding of PNA was observed on murine platelets, increased binding of all the lectins to human platelets occurred following anti-GPIb $\alpha$ mAbs incubations (Supplementary Fig. 2). To confirm that desialylation is a direct consequence of antibody binding, we measured anti-GPIb $\alpha$-mediated desialylation in the presence of 2-deoxy2,3-didehydro- $N$-acetylneuraminic acid (DANA), a synthetic mammalian and bacterial/viral pan-sialidase inhibitor, which possesses a reported half-maximal inhibitory concentration of $\sim 80 \mu \mathrm{M}$ against mammalian neuraminidase- 1 (NEU1) ${ }^{37}$. DANA was found to significantly decrease anti-GPIb $\alpha$-mediated desialylation of murine and human platelets (Fig. 2d,g). In addition, DANA had no effect on desialylation of $G P I b \alpha^{-/-}$ platelets treated with anti-GPIb $\alpha$ mAbs (Supplementary Fig. 3).

In contrast, although anti-GPIIbIIIa mAbs did not significantly affect desialylation on murine platelets, clones 9D2, M1 and HUTA B did cause desialylation on platelets from certain individual donors (Fig. 2e). Similar to what was observed in platelet activation, desialylation caused by anti-GPIIbIIIa mAbs (9D2, M1 and HUTA B) was Fc $\gamma$ RIIa dependent, as blocking with IV.3 completely attenuated the response (Fig. 2h). Importantly, when healthy human platelets were incubated with plasma from ITP patients, anti-GPIb $\alpha$ ITP plasma triggered significant RCA-1 binding, while anti-GPIIbIIIa ITP plasma-induced RCA-1 binding was moderate (Fig. 2i).

Together these results confirm that increased sialidase activity resulting in desialylation directly follows from anti-GPIb $\alpha$ antibody binding. Thus, platelet desialylation may be an important biomarker for platelet activation and for anti-GPIb-mediated ITP.

The GPIb $\alpha$ subunit is predominantly desialylated. Given that GPIb $\alpha$ is the most heavily glycosylated subunit on the platelet surface $^{38}$, we suspected that GPIb $\alpha$ may be the principle target of desialylation. To test this, we utilized $O$-sialoglycoprotein endopeptidase (OSGE) to selectively remove the extracellular $\mathrm{N}$ terminus of GPIb $\alpha^{39}$. Evaluation of RCA-1 binding before and after OSGE treatment revealed that binding decreased to almost baseline levels following removal of GPIb $\alpha$ (Fig. 2j). In addition, following anti-GPIb $\alpha \mathrm{mAb}$ treatment, platelet lysate was blotted with RCA-1 and a commercial anti-GPIb $\alpha$ antibody that identified a polypeptide corresponding to around $150 \mathrm{kDa}$. Western blotting confirmed RCA-1 binding was strongest on

Table 1 | Characterization of mouse anti-mouse mAb against GPIb $\alpha$ and GPIIla.

\begin{tabular}{|c|c|c|c|c|c|}
\hline Clone name & IgG subtype & Antigen & $\begin{array}{c}\text { Species cross-reactivity } \\
\text { (tested) }\end{array}$ & $\begin{array}{c}\text { Inhibits murine } \\
\text { platelet aggregation }\end{array}$ & $\begin{array}{c}\text { Inhibits human platelet } \\
\text { aggregation }\end{array}$ \\
\hline NIT A & $\operatorname{lgG} 2 \mathbf{a}$ & GPIb $\alpha$ & $h, m, p$ & Yes & Yes \\
\hline NIT B & $\operatorname{lgG} 2 \mathbf{a}$ & GPIb $\alpha$ & $h, m, p$ & Yes & Yes \\
\hline NIT F & IgG 2a & GPIb $\alpha$ & $h, m, p$ & Yes & Yes \\
\hline NIT E & $\lg G 2 b$ & GPIb $\alpha$ & $\mathrm{m}$ & Yes & NA \\
\hline NIT G & $\lg G 1$ & GPIb $\alpha$ & $\mathrm{m}$ & Yes & NA \\
\hline NIT H & $\lg G 1$ & GPIb $\alpha$ & $\mathrm{m}$ & Yes & NA \\
\hline 9D2 & IgG 1 & GPIIbIIla & $h, m, p, r$ & Yes & No \\
\hline M1 & IgG 1 & GPIIbIIla & $h, m, p, r$ & Yes & Yes \\
\hline PSI B1 & IgG 1 & GPIIla (PSI domain) & $h, m, p$, rib & Yes & Yes \\
\hline PSI C1 & IgG 1 & GPIIla (PSI domain) & $h, m, p$, rib & Yes & Yes \\
\hline PSI E1 & lgG 2b & GPIIla (PSI domain) & $h, m, p$, rib & Yes & Yes \\
\hline HUTA A & IgG 2a & GPIIbllla & $\mathbf{h}$ & NA & Yes \\
\hline HUTA B & IgG 1 & GPIIbllla & $\mathbf{h}, \mathbf{m}, \mathbf{p}$ & No & Yes \\
\hline
\end{tabular}

IgG, immunoglobulin G; mAb, monoclonal antibody; NA, not applicable.

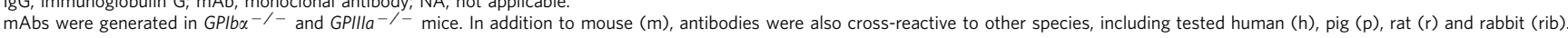
Antibodies cross-reactive with human antigens are in bold. ADP $(20 \mu \mathrm{M})$ - or thrombin (1U)-induced human/mouse platelet aggregation was inhibited by most anti-GPIIbllla mAbs. Ristocetin

$\left(20 \mu \mathrm{gl}^{-1}\right)$-/botrocetin $\left(1.5 \mathrm{mg} \mathrm{ml}^{-1}\right)$-induced human/mouse platelet aggregation was inhibited by anti-GPlb $\alpha \mathrm{mAbs}$. 

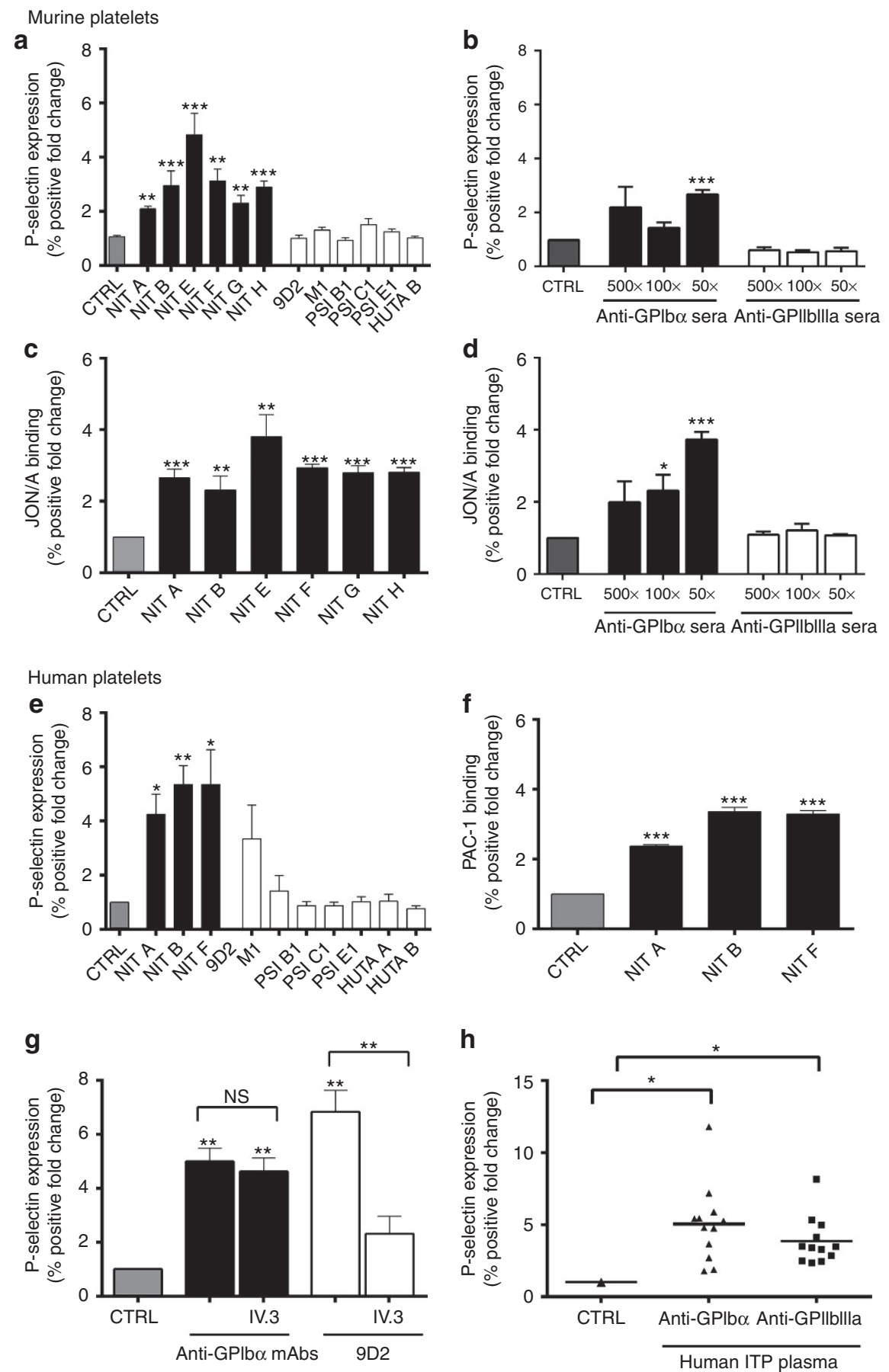

Figure 1 | Anti-GPIb $\boldsymbol{\alpha}$ antibodies induce platelet activation. Surface P-selectin expression on murine (a,b) or human platelets (e,g,h) and JON/A binding on murine $(\mathbf{c}, \mathbf{d})$ or PAC-1 binding on human platelets $(\mathbf{f})$ were measured by flow cytometry following incubation with mAbs $(\mathbf{a}, \mathbf{c}, \mathbf{e}, \mathbf{f}, \mathbf{g})$ or antisera $(\mathbf{b}, \mathbf{d})$. (g) Fc $\gamma$ RIla/III blocker IV.3 was incubated with mAb 9D2 and platelets, and platelet P-selectin was assessed following. Only the healthy donor platelets that were significantly activated in the presence of 9D2 were tested; $n=3$. (h) P-selectin expression was measured in healthy human platelets following incubation with anti-GPIb $\alpha$ (ITP-1 to ITP-12) or anti-GPIIbIlla (ITP-a to ITP-I) antibody-positive ITP patient plasma. All flow cytometry data are expressed as fold change from nonspecific murine IgG (murine)- or IVIG (human)-treated control platelets (CTRL). Anti-GPIb $\alpha$ mAbs shown as mean \pm s.e.m. of individual mAbs. ${ }^{\star} P<0.05,{ }^{\star \star} P<0.01,{ }^{\star \star \star} P<0.001$ versus $C T R L$ as analysed by the Student's $t$-test (a-f) or one-way analysis of variance followed by Bonferroni post hoc analysis (g,h). NS, not significant.

GPIb $\alpha$ and was susceptible to DANA inhibition (Fig. 2k). These data indicate that GPIb $\alpha$ is likely the dominant target of anti-GPIb $\alpha$-mediated desialylation.

Anti-GPIb $\alpha$ antibodies induce surface expression of NEU1. Since sialidase NEU1 has been reported to be involved in platelet desialylation ${ }^{40}$, we established a method to detect surface platelet NEU1 using flow cytometry, and found that anti-GPIb $\alpha$, but not anti-GPIIbIIIa or isotype control antibodies, induced NEU1 translocation onto both murine and human non-permeabilized platelets (Fig. 3a-f; Supplementary Fig. 4). This NEU1 was found to be abundant in the granules of permeabilized platelets (Fig. 3d). Consistent with our RCA-1-binding results (Fig. 2b,f), 
Murine platelets
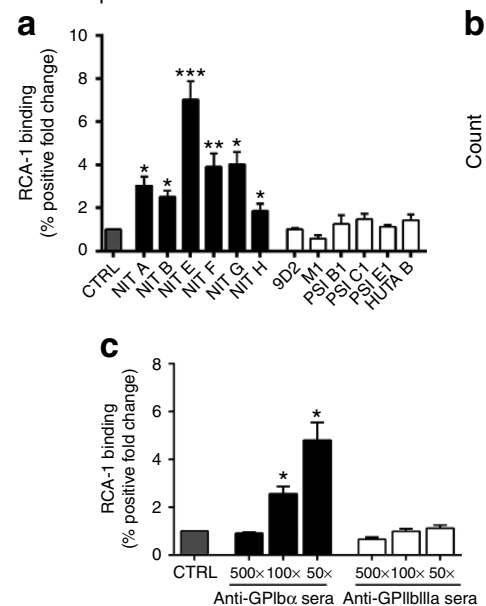

b
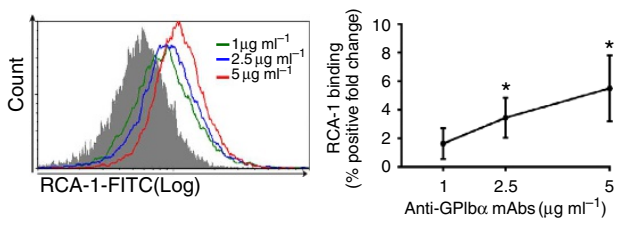
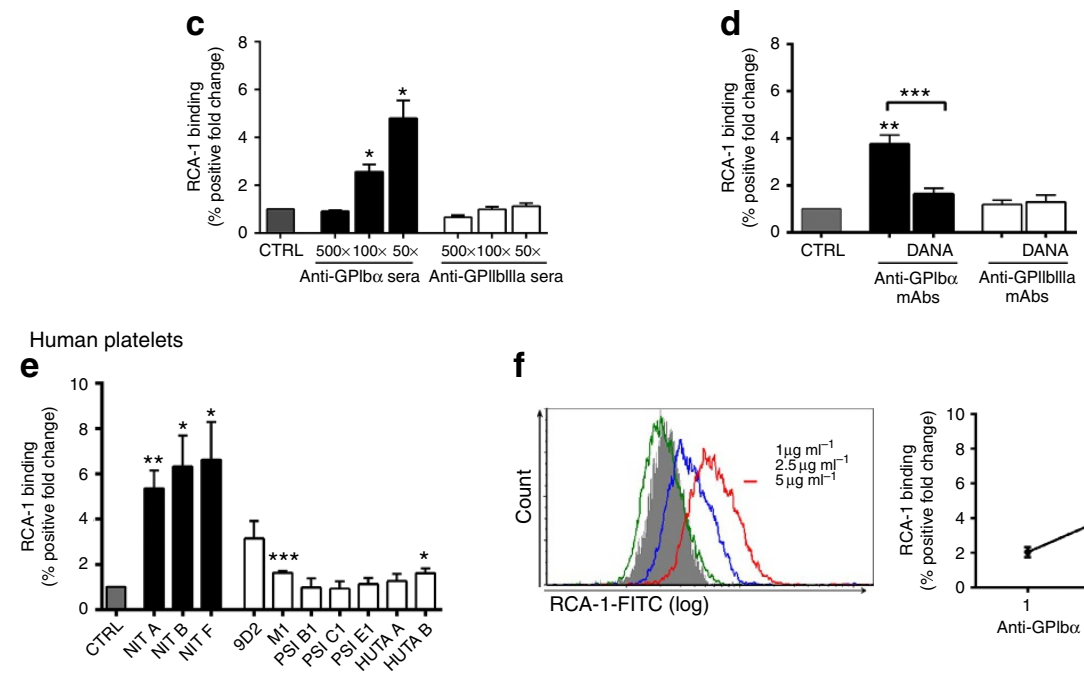

f

g

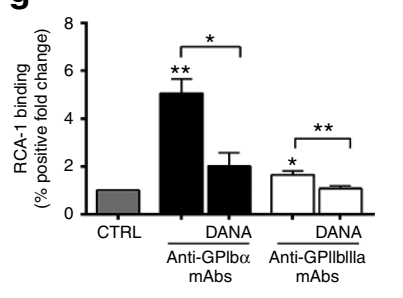

j

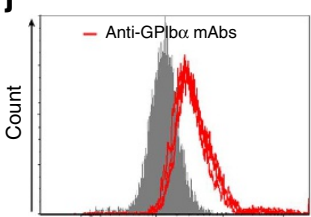

RCA-1-FITC(Log)

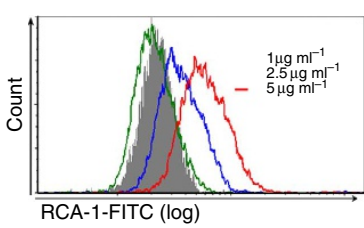

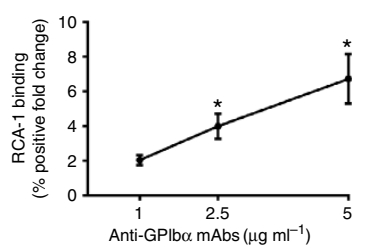

i

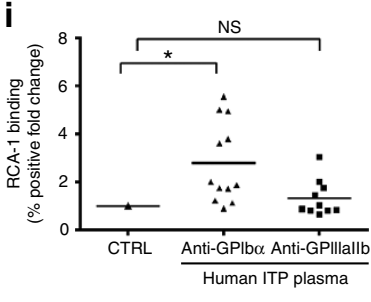

h

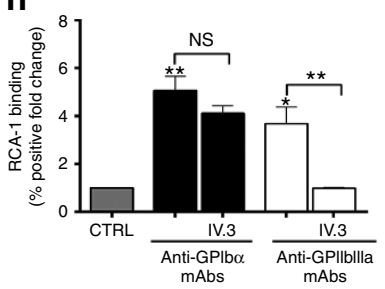

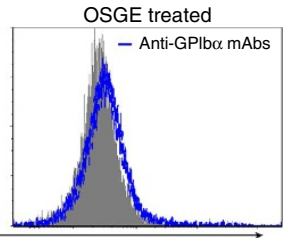

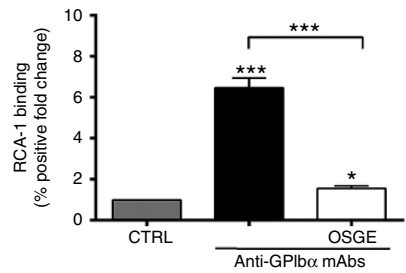

$\mathbf{k}$
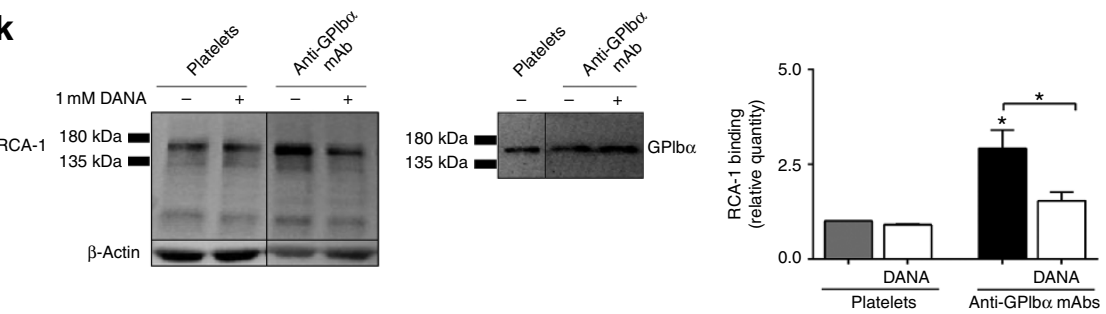

Figure 2 | Antibody-mediated platelet desialylation occurs mainly on the GPIb $\boldsymbol{\alpha}$ subunit. Galactose exposure was detected with RCA-1 binding and measured by flow cytometry in murine (a-d) and human $(\mathbf{e}-\mathbf{j})$ platelets following incubation with $\mathrm{mAbs}(\mathbf{a}, \mathbf{b}, \mathbf{d}, \mathbf{e}, \mathbf{f}-\mathbf{h}, \mathbf{j}, \mathbf{k})$ or antisera $(\mathbf{c}) n=10-20$. $(\mathbf{d}, \mathbf{g})$ Co-incubation with sialidase inhibitor DANA prior to addition of mAbs to murine (d) or human ( $(\mathbf{g})$ platelets; $n=8$. (h) Fc $\mathrm{RII} / \mathrm{III}$ blocker IV.3 was incubated with mAbs (9D2, M1 or HUTA B) and platelets, RCA-1 binding was assessed following. Only the healthy donor platelets that were significantly desialylated in the presence of these mAbs were tested; $n=4$. (i) RCA-1 binding was measured in healthy human platelets following incubation with anti-GPIb $\alpha$ (ITP-1 to ITP-12) or anti-GPIIbllla (ITP-a to ITP-I) antibody-positive ITP patient plasma. (j) anti-GPIb $\alpha$-mediated RCA-1 binding was assessed following removal of GPIb $\alpha$ with OSGE. (k) Representative western blot of RCA-1 binding (left), and probing with commercial anti-GPIb $\alpha$ antibody (right) to confirm the identity of the RCA-1-positive band following incubation with anti-GPIb $\alpha$ mAb (NIT F). RCA-1 binding on GPIb $\alpha$ was also quantified by protein densitometry in the presence of DANA. All flow cytometry data are expressed as fold change from nonspecific murine IgG (murine)- or IVIG (human)-treated control platelets (CTRL). Anti-GPlb $\alpha$ mAbs shown as mean \pm s.e.m. of individual mAbs. ${ }^{\star} P<0.05,{ }^{\star \star} P<0.01$, ${ }^{\star \star \star} P<0.001$ versus CTRL as analysed by the Student's $t$-test $(\mathbf{a}-\mathbf{c}, \mathbf{e}, \mathbf{f})$ or one-way analysis of variance followed by Bonferroni post hoc (d,g-k). NS, not significant. 
we observed a dose-dependent increase of NEU1 translocation with increasing concentrations of anti-GPIb $\alpha \mathrm{mAb}$ (Fig. 3c,f). Triple staining of P-selectin, RCA-1 and NEU1 on platelets following anti-GPIb $\alpha \mathrm{mAb}$ incubations revealed significant coexpression and correlation between these markers within the same platelet population $(P<0.001$ as assessed by Pearson's correlation, Supplementary Figs 5 and 6). These data not only strongly implicate NEU1 as the enzyme mediating desialylation, but also link desialylation with platelet activation.

Platelet activation and desialylation positive feedback loop. We next determined whether the observed anti-GPIb $\alpha$ antibody effects were acting through canonical GPIb $\alpha$ activation signalling cascades. We first tested whether various inhibitors of known downstream GPIb $\alpha$ activation signalling molecules could decrease anti-GPIb $\alpha \mathrm{mAb}$-mediated platelet activation. We found inhibitors of intracellular $\mathrm{Ca}^{2}+$ flux (BAPTA-AM), P38MAPK activity (SB203580) and Src kinases (PP1) all significantly decreased anti-GPIb $\alpha$ antibody-induced human platelet activation as measured by $\mathrm{P}$-selectin, while Q-VD-OPh, a pan caspase inhibitor of platelet apoptosis did not appear to have any effect (Fig. 4a). Western blot analysis further demonstrated anti-GPIb $\alpha$ $\mathrm{mAb}$ can directly mediate GPIb $\alpha$ downstream signalling as evidenced by increased phosphorylation of p38MAPK (Fig. 4b), which could be inhibited by SB203580 and PP1 as quantified by densitometry (Fig. 4b,c). These data indicate that anti-GPIb $\alpha$ antibodies directly mediate platelet activation through endogenous GPIb $\alpha$ signalling pathways.

To further elucidate the link between anti-GPIb $\alpha$-mediated platelet activation and desialylation, we first tested whether inhibitors of platelet activation may affect platelet desialylation. a Murine platelets
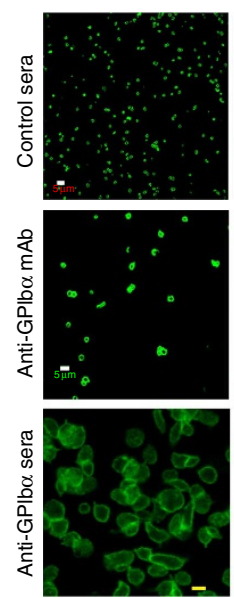

Anti-CD61

d Human platelets

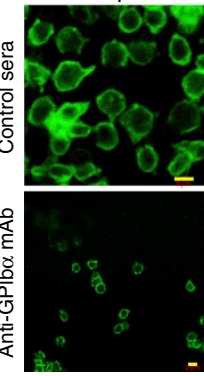

Anti-CD61
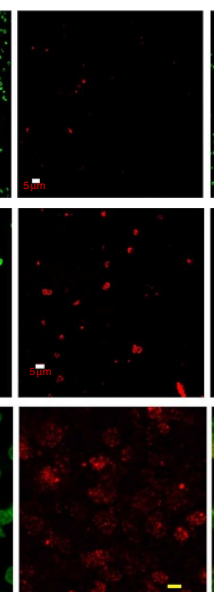

Anti-NEU1

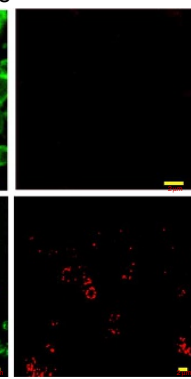

Anti-NEU1
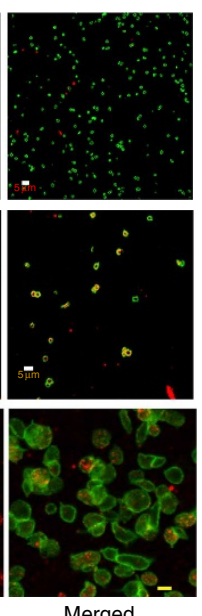

C

b
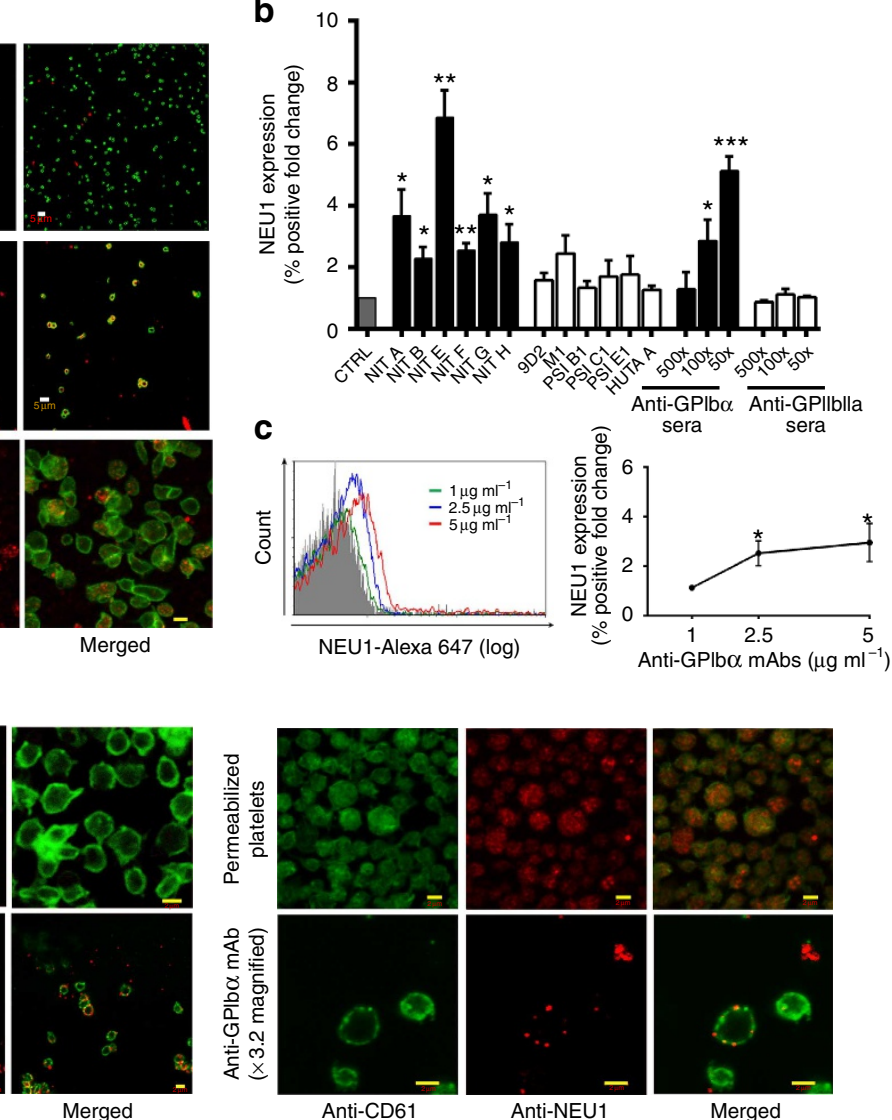

f
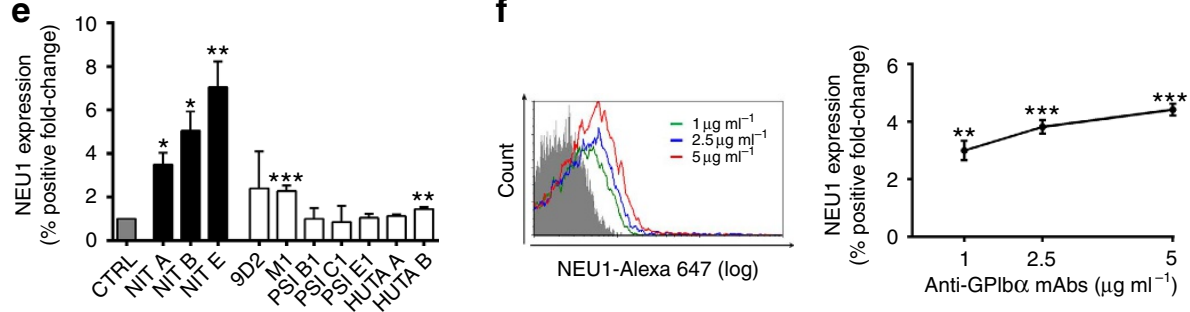

Figure 3 | Anti-GPIb $\boldsymbol{\alpha}$ antibodies induce surface expression of NEU1. Representative confocal images of surface expression of NEU1 on murine (a) and human (d) platelets stained with anti-NEU1 and anti-CD61 following incubations with anti-GPIb $\alpha$ mAb (NIT G (murine), NIT B (human)) or anti-GPIb $\alpha$ sera (murine). All other mAbs were also tested with similar results; $n=5-8$. Total NEU1 was detected in permeabilized human platelets; $n=2$. White scale bars, $5 \mu \mathrm{M}$; yellow scale bars, $2 \mu \mathrm{M}$. (b,c,e,f) Flow cytometric analysis of surface NEU1 expression on murine (b,c) and human (e,f) platelets following incubations with anti-GPIb $\alpha$ mAb or sera. Anti-GPIb $\alpha$ mAbs shown as mean \pm s.e.m. of individual mAbs; $n=5-8$. All flow cytometry data are expressed as fold change from nonspecific murine IgG (murine)- or IVIG (human)-treated control platelets (CTRL). Anti-GPIb $\alpha$ mAbs shown as mean \pm s.e.m. of individual mAbs. ${ }^{\star} P<0.05,{ }^{\star \star} P<0.01,{ }^{\star \star \star} P<0.001$ versus CTRL as analysed by the Student's $t$-test. 
a
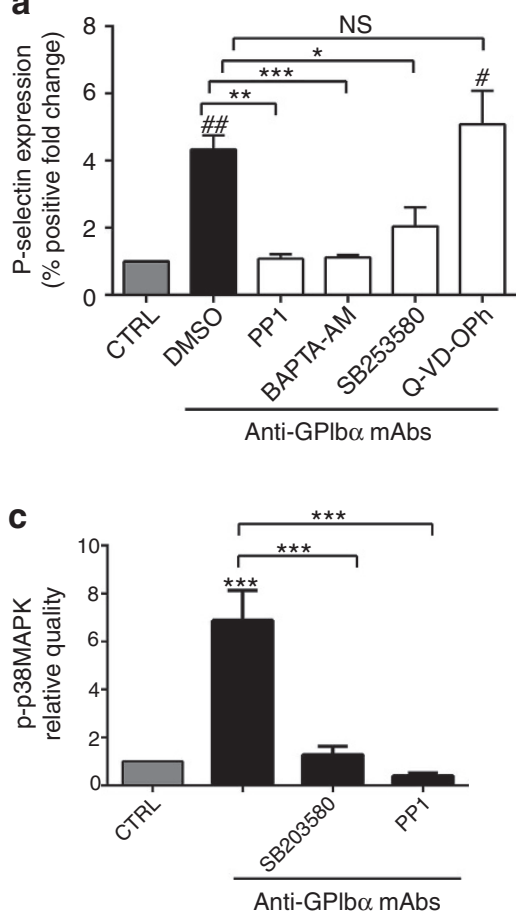

b

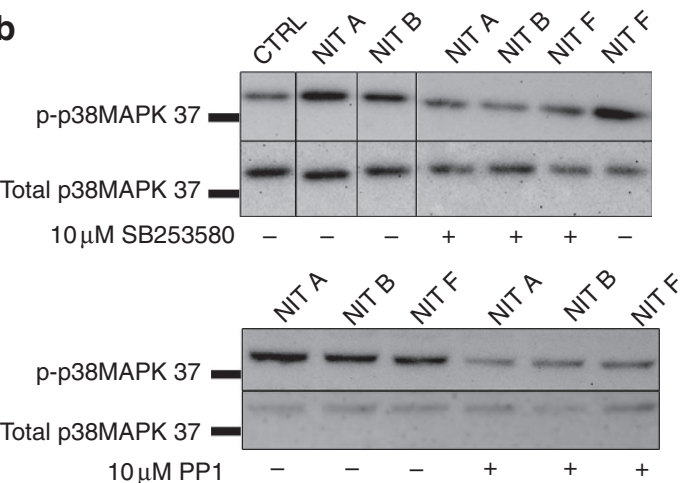

d
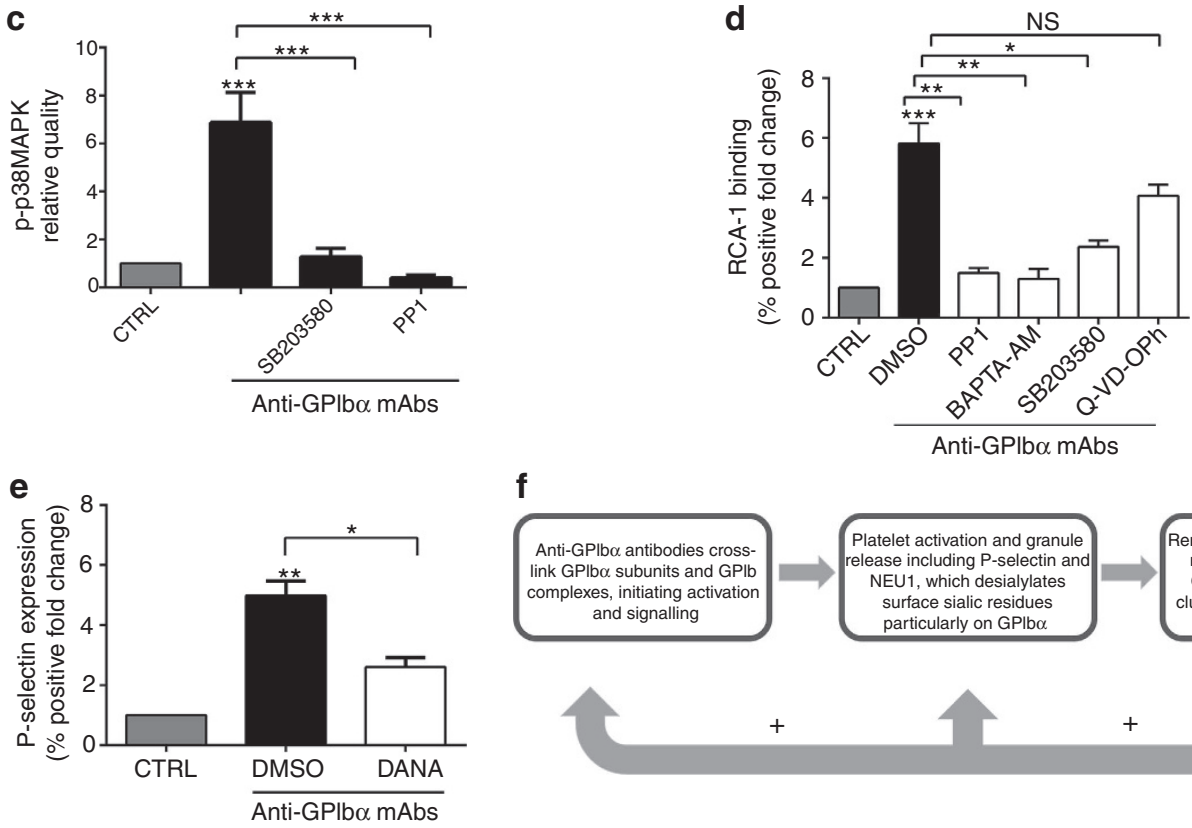

f
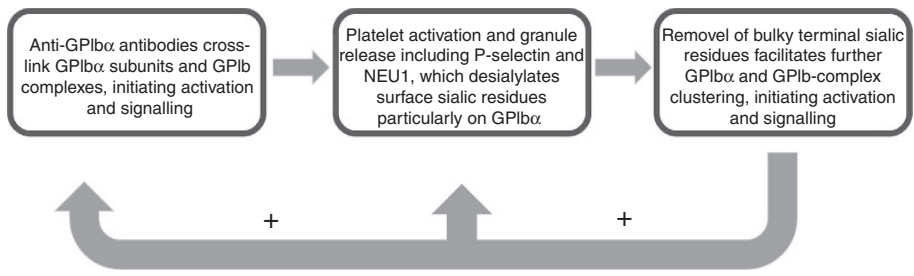

g Murine platelets
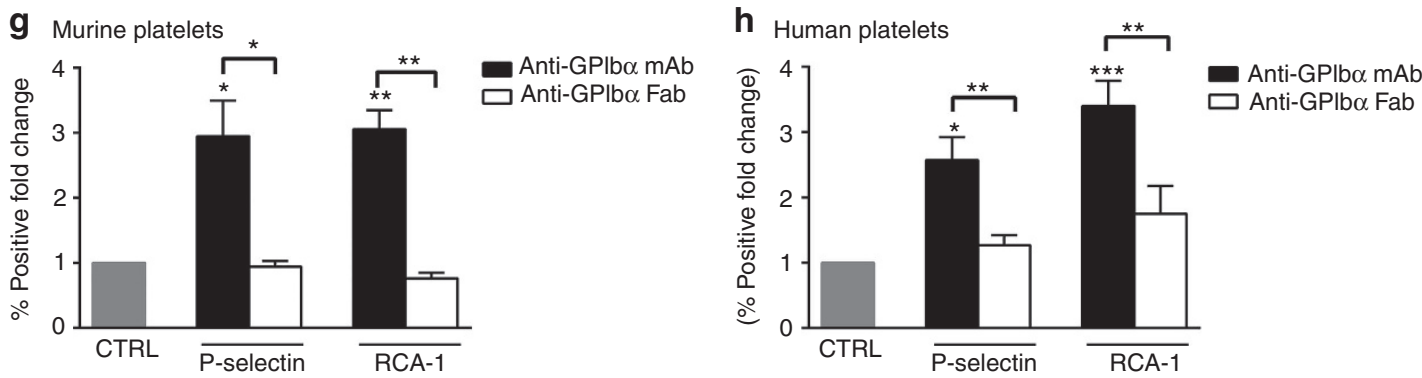

Figure 4 | Anti-GPIb $\alpha$ platelet activation and desialylation is a positive feedback loop. (a,d) Human platelets were pre-incubated with inhibitors of platelet activation including intracellular $\mathrm{Ca}^{2+}$ flux (BAPTA-AM), phosphorylation of P38MAPK (SB203580) and Src kinase (PP1) prior to addition of anti-GPIb $\alpha$ mAbs. Following which, platelet activation (P-selectin expression) (a) or desialylation (RCA-1 binding) (d) was detected via flow cytometry. \# and \#\# indicate comparison with CTRL for a only; $n=8$. (b) Representative western blots of whole-platelet lysate following incubations with GPIb $\alpha$ mAbs with or without indicated inhibitors. Membranes were probed for phosphorylated p38MAPK ( $p$-p38MAPK) then stripped and re-probed for total pMAP38K. (c) Densitometry protein quantification of p-P38MAPK detected in $\mathbf{b}$. Data representative of three separate experiments. (e) Sialidase inhibitor DANA was added prior to anti-GPIb $\alpha$ mAb incubation. DANA-mediated inhibition of antibody-induced platelet activation was measured by P-selectin expression and detected via flow cytometry. $n=6$. (f) Schematic flow chart illustrating platelet activation-desialylation-positive feedback loop. Following initial anti-GPIb $\alpha$ antibody crosslinking of GPIb $\alpha$ subunits, activation signalling occurs leading to surface translocation of NEU1. NEU1 cleavage of terminal sialic residues on a GPIb $\alpha$ subunit facilitates receptor clustering, resulting in amplification of platelet activation. (g,h) NIT A, NIT B and NIT F Fab fragments were generated and their effects on platelet activation and desialylation on murine $(\mathbf{g})$ and human platelets $(\mathbf{h})$ were analysed by flow cytometry. $n=5$. All flow cytometry data are expressed as fold change from nonspecific murine IgG (murine)- or IVIG (human)-treated control platelets (CTRL), unless otherwise indicated. Anti-GPIb $\alpha$ mAbs shown as mean \pm s.e.m. of individual mAbs. ${ }^{\star}, \# P<0.05,{ }^{\star \star}, \# \# P<0.01,{ }^{\star \star \star} P<0.001$ as assessed by one-way analysis of variance followed by Bonferroni post hoc analysis. NS, not significant. 
We found inhibitors of GPIb $\alpha$ activation SB203580, BAPTA-AM and PP1, but not platelet apoptosis (Q-VD-OPh), markedly inhibited anti-GPIb $\alpha \mathrm{mAb}$-induced desialylation (Fig. 4d). This indicates that GPIb $\alpha$ activation is a prerequisite for anti-GPIb $\alpha$ mAb-mediated desialylation. Conversely, to test whether desialylation had any effect on platelet activation, we treated platelets with the sialidase inhibitor DANA in the presence of anti-GPIb $\alpha$ mAbs. DANA strongly inhibited anti-GPIb $\alpha$-induced P-selectin expression (Fig. 4e). Together, these data establish that, while GPIb $\alpha$ activation may be required for desialylation, desialylation itself may further potentiate platelet activation. It has been previously reported that removal of bulky terminal sialic residues can facilitate GPIb-receptor clustering and outsidein signalling ${ }^{41,42}$. Thus, anti-GPIb $\alpha$ antibody binding, which initiates platelet activation, NEU1 translocation and GPIb $\alpha$ desialylation, creates a positive feedback loop culminating in additional P-selectin expression and platelet desialylation (Fig. 4f).

Since GPIb $\alpha$ clustering has been linked to platelet signalling and activation ${ }^{43,44}$, we generated Fab monovalent antibody fragments from several anti-GPIb $\alpha$ mAbs. Fab fragments incubated with both human and murine platelets did not cause significant platelet activation or desialylation (Fig. 4g,h). We thus propose that GPIb $\alpha$ antibody crosslinking is fundamental for antibody-mediated platelet activation and desialylation.

Platelet activation and desialylation occur in vivo. To test whether our in vitro observations occur in vivo, anti-GPIb $\alpha$ and anti-GPIIbIIIa mAbs or antisera were injected into female $\mathrm{BALB} / \mathrm{c}$ mice to induce thrombocytopenia. Platelets isolated from this model $16 \mathrm{~h}$ post injection were co-stained for anti-mouse IgG and RCA-1 or P-selectin to assess the percentage of anti-GPIb $\alpha$ antibody-bound platelets that were positive for platelet activation and desialylation. We found that in NIT A-, NIT B- and NIT F-injected mice, $>50 \%$ of antibody-bound platelets were desialylated, while NIT E-, NIT G- and NIT H-injected mice ranged from 20 to $40 \%$. In addition, there was a significant proportion of antibody-bound platelets positive for P-selectin expression, albeit at lower percentages than desialylation (Fig. 5a,b). In contrast, platelets from thrombocytopenic mice injected with anti-GPIIbIIIa $\mathrm{mAb}$ or antisera did not exhibit these effects (Fig. 5b). Overall, in vivo antibody-mediated platelet activation and desialylation were observed to be more pronounced than in vitro. Thus, anti-GPIb $\alpha$ antibodies induced significant increases in platelet activation and desialylation in a clinically relevant model of ITP.

Anti-GPIb $\alpha$-opsonized platelets can be cleared via the AMR. The Ashwell-Morell receptor (AMR), an asialoglycoprotein counter receptor predominantly expressed on hepatocytes, was reported to mediate clearance of desialylated $\mathrm{GPs}^{45}$, and has been linked to maintenance of normal platelet turnover through the platelet deglycosylation state ${ }^{24,46}$. We suspected that the AMR may contribute to the destruction of the antibody-mediated desialylated platelets. To test this, we inhibited the AMR using asialofetuin and assessed the clearance of anti-GPIb $\alpha$-opsonized platelets. We found that following co-injection of anti-GPIb $\alpha$ opsonized platelets with asialofetuin, uptake of antibody-bound platelets through the AMR was attenuated, resulting in an increase by $\sim 25 \%$ of circulating anti-GPIb $\alpha$-bound platelets compared with fetuin, a nonspecific control (Fig. 6a). Furthermore, asialofetuin did not rescue anti-GPIIbIIIa $\mathrm{mAb}$ (9D2)- or antisera-opsonized platelets (Fig. 6b).
To further confirm the contribution of the AMR in Fcindependent anti-GPIb $\alpha$ antibody-mediated thrombocytopenia, we first injected anti-GPIb $\alpha \mathrm{mAbs}$ into $F c \gamma R^{-/-}$mice and found thrombocytopenia still occurred (Fig. 6c), particularly when injected with anti-GPIb $\alpha$ mAb NIT G (Supplementary Fig. 7a), while anti-GPIIbIIIa mAbs failed to induce a significant decrease in platelets in these mice (Fig. 6d). We next compared the severity of thrombocytopenia between Ashwell-Morell-deficient (Aspgr $2^{-1-}$ ) and syngeneic WT control mice and found thrombocytopenia in Aspgr2 ${ }^{-/-}$mice was significantly attenuated when injected with the same concentration of anti-GPIb $\alpha$ $\mathrm{mAb}$ (Fig. 6e; Supplementary Fig. 7b), suggesting that the AMR indeed plays a significant role in the clearance of anti-GPIb $\alpha$ bound platelets.

The importance of the AMR in clearance of anti-GPIb $\alpha$ opsonized platelets was further substantiated in macrophagedepleted mice. We found macrophage-depleted mice still maintained clearance of anti-GPIb $\alpha$-opsonized platelets from circulation, while clearance of anti-GPIIbIIIa-opsonized platelets was almost completely rescued. In addition, co-injections of asialofetuin almost completely inhibited anti-GPIb $\alpha$-mediated platelet clearance (Fig. 7a). This suggests that the role of the AMR in anti-GPIb $\alpha$-antibody-mediated platelet clearance is dominant in the absence of macrophages.

Tissue sections of the spleen and liver revealed significant co-localization of anti-GPIb $\alpha$-opsonized platelets in the liver, but not in the spleen of macrophage-depleted mice, $(P<0.05$ as determined by the Student's $t$-test, Fig. $7 \mathrm{c}-\mathrm{e})$. Treatment with asialofetuin resulted in decreased platelet localization in the livers of macrophage-depleted mice (Fig. 7c). Although asialofetuin did not decrease platelet localization in WT livers, the fluorescent platelets do not appear to co-localize with AMRs (Fig. 7b), suggesting that they may be binding to other phagocytic cells (for example, Kupffer macrophages).

These findings indicate for the first time that the AMR on hepatocytes significantly contributes to the clearance of antiGPIb $\alpha$ mAb-opsonized platelets, particularly in the absence of macrophages. Thus, hepatocytes play a previously unappreciated and important role in platelet clearance in antibody-mediated thrombocytopenia.

Sialidase inhibition can attenuate ITP. Patients who test positive for anti-GPIb $\alpha$ antibodies tend to be more refractory to standard first-line treatments, such as steroids and $\mathrm{IVIG}^{13,14,16}$. We explored the therapeutic potential of sialidase inhibitors in our murine ITP model. We found that pretreatment with DANA significantly decreased desialylation of platelets in anti-GPIb $\alpha$, but not anti-GPIIbIIIa mAb-injected mice (Fig. 8a). Remarkably, DANA also rescued platelet counts in most of the anti-GPIb $\alpha$ mAbs tested (NIT B, NIT E, NIT F, NIT G and NIT H) and antiGPIb $\alpha$-sera-injected mice (Fig. 8b,c). As expected, DANA did not significantly increase platelet counts in anti-GPIIbIIIa mAb- or sera-injected mice (Fig. 8c; Supplementary Fig. 8). We then tested the therapeutic potential of oseltamivir phosphate (Tamiflu), a commonly administered anti-influenza medication that inhibits viral neuraminidase, and found significant amelioration of platelet counts in our murine model of anti-GPIb $\alpha$-mediated ITP (Fig. 8d). In conclusion, this is the first study in mice to provide evidence to support utilization of sialidase inhibitors as a viable therapeutic alternative for refractory ITP patients.

\section{Discussion}

We describe herein a novel Fc $\gamma \mathrm{R}$-independent mechanism of platelet clearance in thrombocytopenia mediated predominantly by anti-GPIb $\alpha$ antibodies, which is distinct from Fc-dependent 


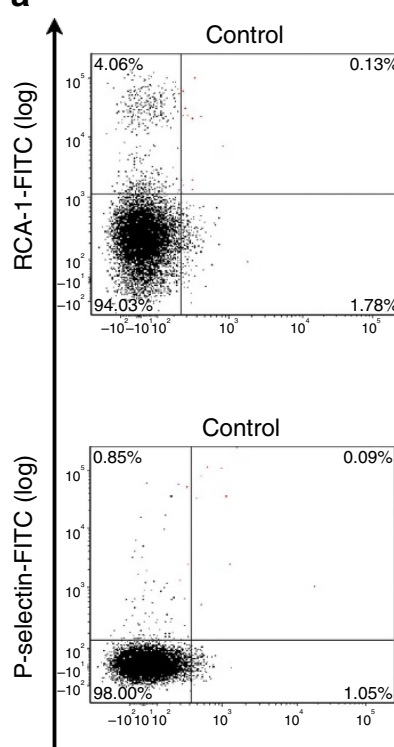

RCA-1
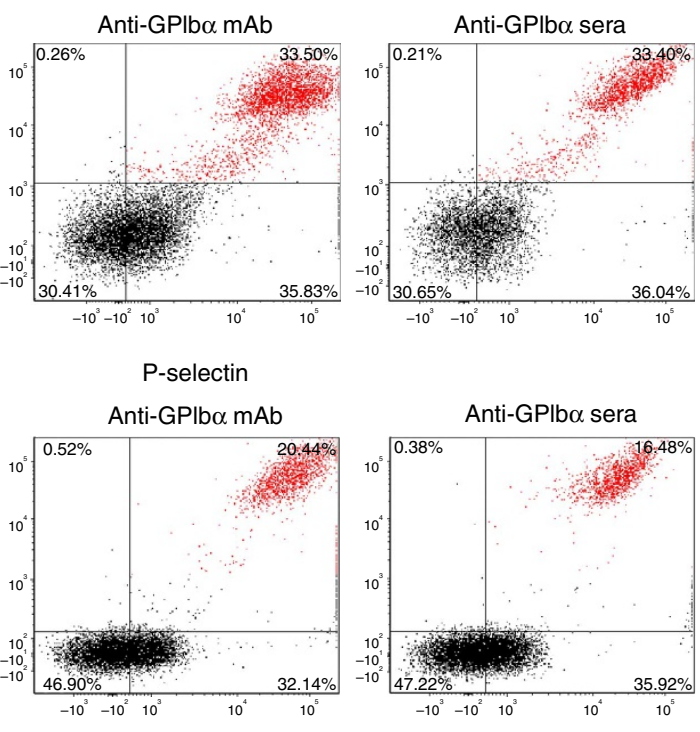

Anti-mouse lgG-Alexa $647(\log )$

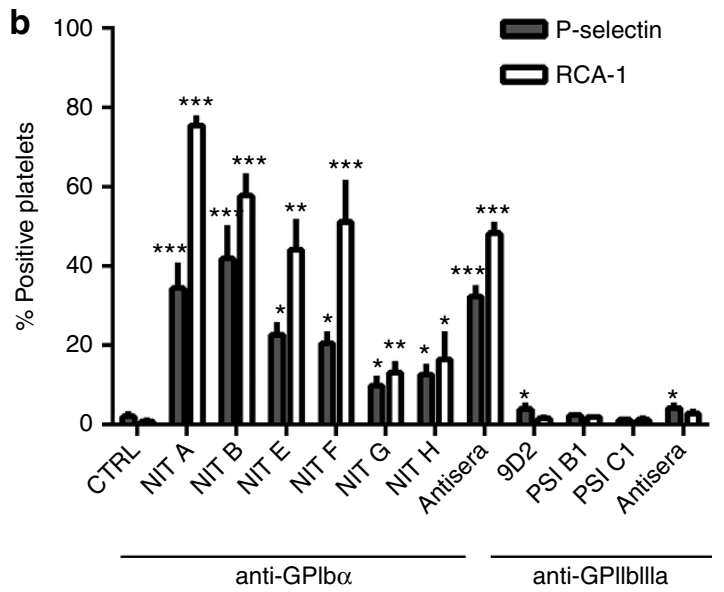

Figure 5 | Anti-GPIb $\boldsymbol{\alpha} \mathbf{m A b s}$ induce platelet activation and desialylation in vivo. (a,b) ITP was induced by intraperitoneal injection of anti-GPIb $\alpha \mathrm{mAbs}$ (NIT A, NIT B, NIT E, NIT F, NIT G and NIT H) or sera. At $16 \mathrm{~h}$ post injection, platelets isolated from the mice were examined for activation (P-selectin) and desialylation (RCA-1) by flow cytometry; $n=3$ per mAb. (a) Representative dot plots of isolated washed platelets from anti-GPIb $\alpha$ mAb (NIT E)- and serainjected mice double-stained for anti-mouse IgG (against anti-GPIb $\alpha$ antibodies) ( $x$ axis) and RCA-1/P-selectin ( $y$ axis).

ITP in both mechanism and therapeutic management. We demonstrate both in vitro and in vivo that binding of mAbs to GPIb $\alpha$ induced significant platelet activation, surface NEU1 translocation and platelet desialylation. Importantly, we found that ITP patient plasma containing anti-GPIb $\alpha$ antibodies induced similar effects on healthy human platelets. This novel mechanism led to increased anti-GPIb $\alpha$-mediated platelet sequestration in the liver and involvement of the hepatocyte in the phagocytosis of platelets in ITP via a previously undescribed pathway. Most importantly, our data demonstrate that sialidase inhibitors can block this pathologic process and ameliorate thrombocytopenia. These findings not only reveal a new mechanism of ITP, but also introduce a potential new diagnostic tool (for example, RCA-1 and NEU1 staining) and a novel therapeutic method (for example, sialidase inhibition), which will have a particularly significant impact on patients refractory to current ITP therapies.

The Fc-independent platelet clearance observed in anti-GPIb $\alpha-$, but not anti-GPIIbIIIa-mediated thrombocytopenia, is most likely due to the distinct effects the antibodies have on their corresponding platelet receptors ${ }^{17,18}$. The ability of anti-GPIb $\alpha$ antibodies to affect platelet signalling has been previously described. We previously observed in murine models of fetal/neonatal alloimmune thrombocytopenia that anti-GPIb $\alpha$ antibodies increased both platelet activation and thrombus formation in the placenta resulting in miscarriage ${ }^{25}$. In addition, several studies have reported that anti-GPIb $\alpha$ antibodies affect platelet function independent of the $\mathrm{Fc}$ region of IgG. Specifically, $\mathrm{F}(\mathrm{ab})_{2}$ fragments of various antiGPIb $\alpha$ antibodies induced platelet agglutination and GPIIbIIIadependent aggregation in vitro ${ }^{30,47}$ and caused thrombocytopenia in vivo when tested as anti-thrombotic agents ${ }^{48,49}$. Here, we found that anti-GPIb $\alpha$ antibodies induced platelet activation via an intracellular signalling cascade typical of von Willebrand factor binding. This led to the previously unobserved antibodymediated desialylation of GPIb $\alpha$. In contrast, anti-GPIIbIIIa antibodies did not induce these effects in murine platelets.

Interestingly, we found differences between murine and human platelet responses to anti-GPIIbIIIa antibodies, whereby anti-GPIIbIIIa mAbs and ITP patient plasma induced activation 

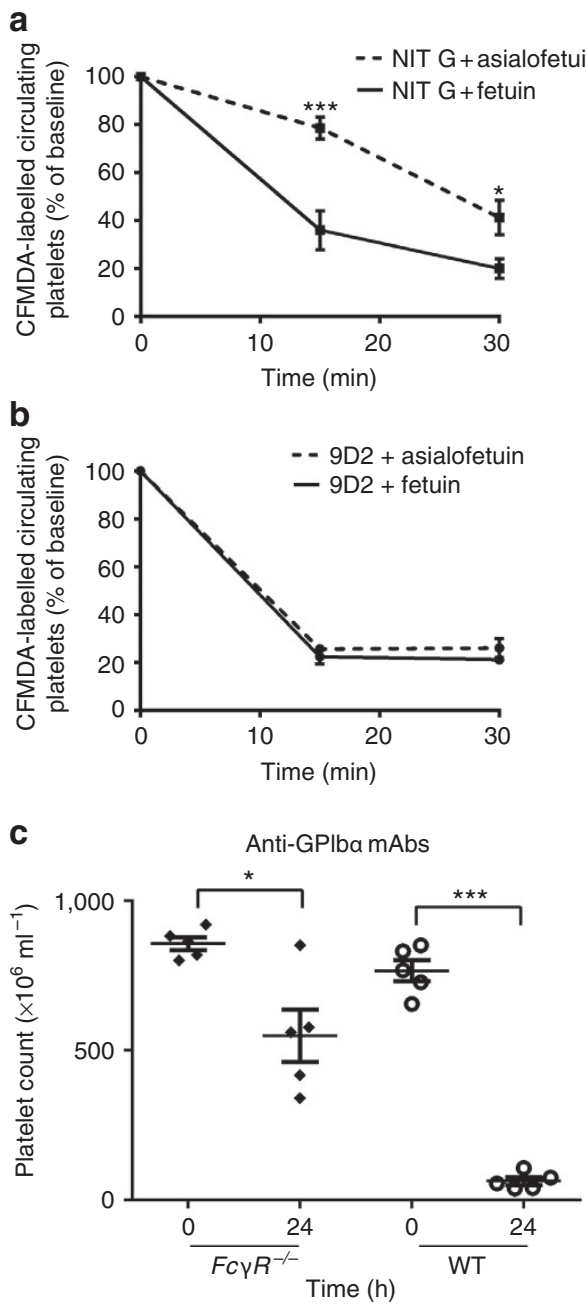

d
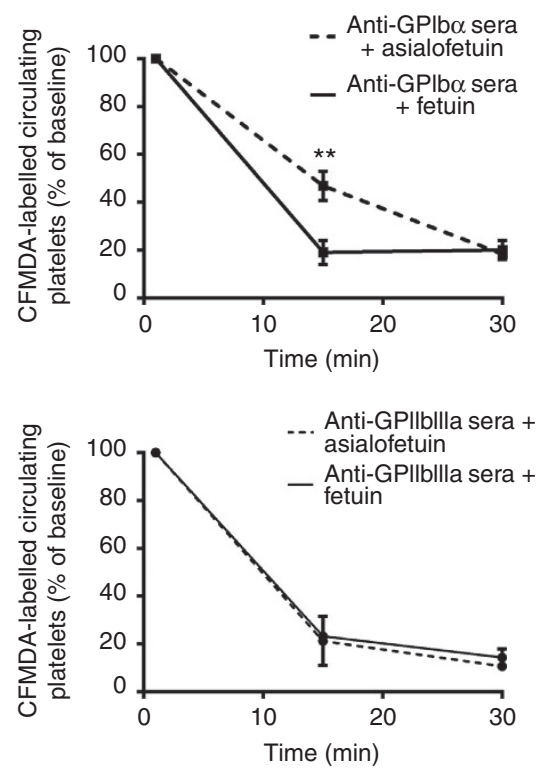

Anti-GPIlbllla mAbs
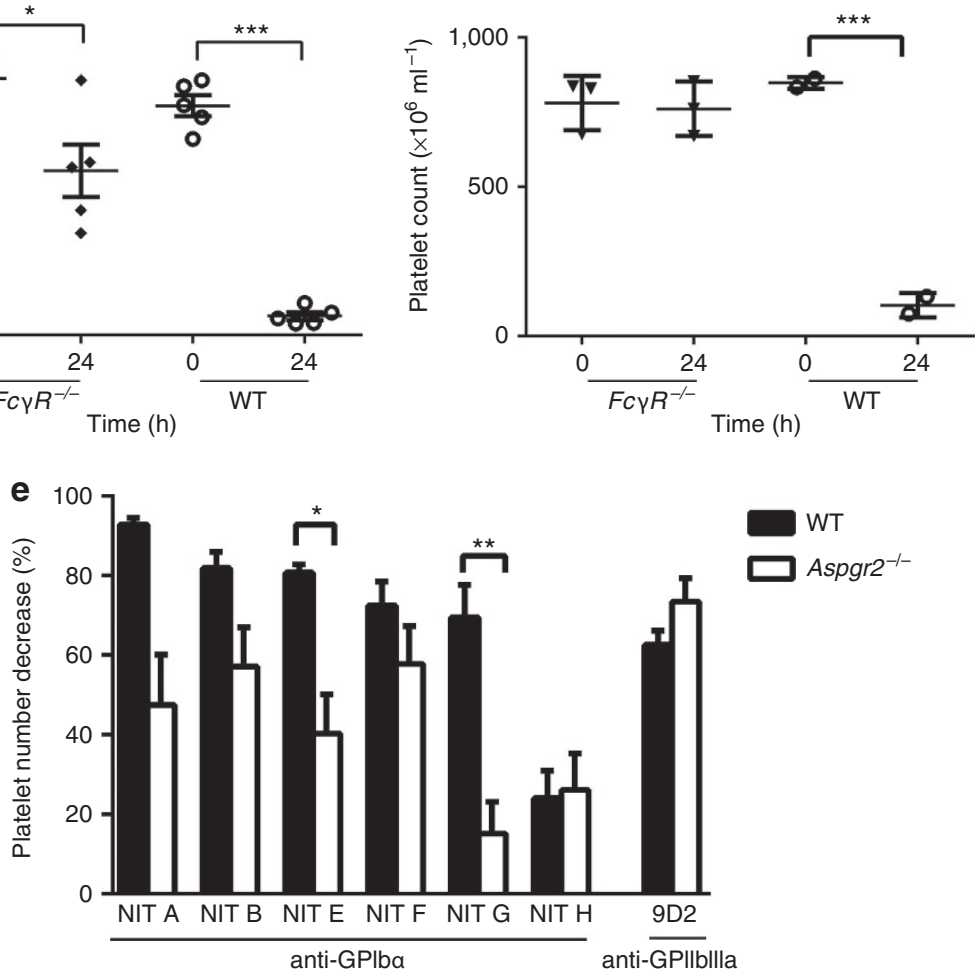

Figure 6 | Fc-independent anti-GPIb $\alpha$-mediated thrombocytopenia occurs via the AMR. Mice were co-injected with (a) anti-GPIb $\alpha$ (NIT G) or (b) anti-GPIIbllla mAb (9D2)- or polyclonal sera-opsonized CFMDA-labelled platelets with asialofetuin (AMR inhibitor) or fetuin (a nonspecific control). Mice were bled at indicated time points and the percentage of fluorescently labelled platelets remaining in circulation was assessed with flow cytometry; $n=6$. ${ }^{\star} P<0.05$ versus Fetuin-injected group at same time point as analysed by two-way analysis of variance followed by Bonferroni post hoc analysis. (c-e) Same doses of indicated mAb or anti-GPIb $\alpha$ or anti-GPllbllla mAbs (equal ratio mixture of individual antibody clones) were injected into age-matched

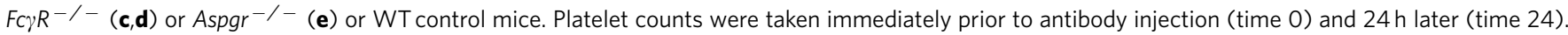
(e) Data are represented as a percentage calculated from [(time 0 platelet count - time 24 platelet count)/time 0 platelet count] $\times 100$. ${ }^{\star} P<0.05$, ${ }^{\star \star} P<0.01,{ }^{\star \star \star} P<0.001$ as determined by the Student's $t$-test.

and desialylation of certain healthy donor platelets. We attributed this response to the low-affinity IgG receptor, Fc $\gamma$ RIIa (the only Fc $\gamma R$ expressed on human but not murine platelets), as IV.3 was shown to attenuate anti-GPIIbIIIa-mediated effects on human platelets. Therefore, it cannot be ruled out that some antibodies targeting GPIIbIIIa may also lead to platelet activation and desialylation, particularly in severe cases of ITP where high antibody titres may be sufficient to induce immune-complex binding via platelet Fc $\gamma$ RIIa. Importantly, IV.3 had no significant effect on anti-GPIb $\alpha$ antibody-mediated human platelet 


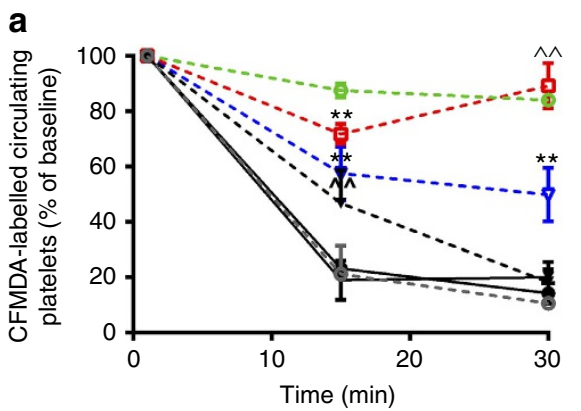

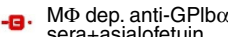
sera+asialofetuin

$-\nabla$. MФ anti-GPIbo

๑. MФ anti-GPIIbIlla sera

* WT anti-GPlb $\alpha+$

fetuin

WT anti-GPIlbllla+

fetuin

Anti-GPIlbllla and

asialofetuin

. Anti-GPlb $\alpha$ and

Time $(\min )$

b Liver
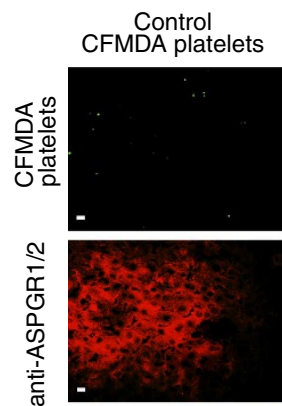

然

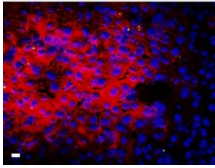

d
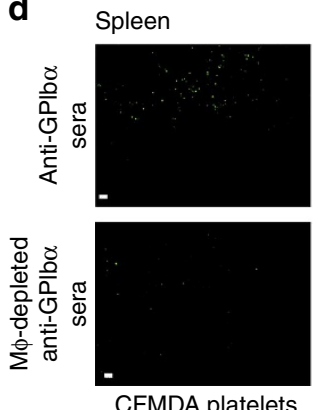

CFMDA platelets
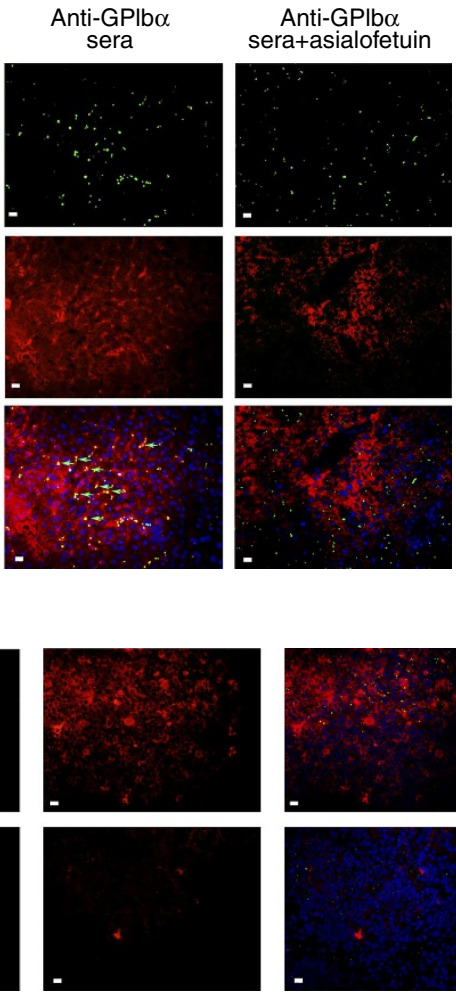

Anti-F4/80
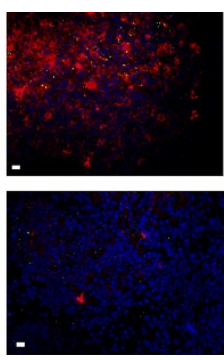

Merged

\section{M $\phi$-depleted mice}

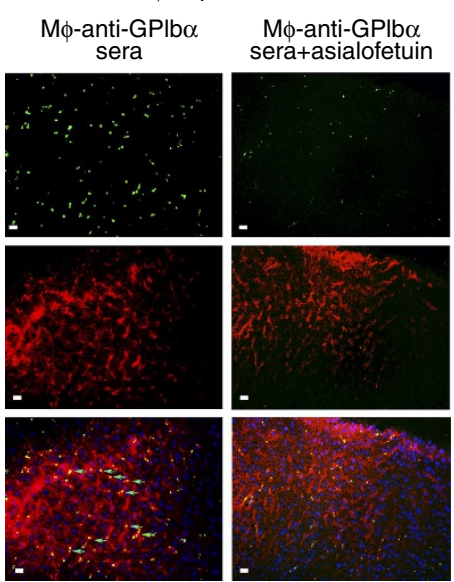

e

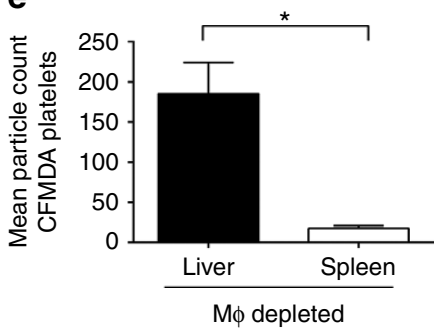

M $\phi$ depleted

Figure 7 | Anti-GPIb $\alpha$ platelet clearance in macrophage-depleted mice is via the AMR. (a-e) Macrophages were depleted with clondrate liposomes via intravenous injection prior to study, and CFMDA-labelled antisera-opsonized platelets were injected with asialofeuin or fetuin and circulating platelets were quantified (a) as described in Fig. 6. $n=5 .{ }^{\wedge} P<0.01$ versus fetuin-injected group at the same time point. ${ }^{\star} P<0.05$, ${ }^{\star \star} P<0.01$ versus baseline as determined by two-way analysis of variance followed by Bonferroni post hoc analysis. (b-d) Tissue sections of the spleen and liver harvested from normal or macrophage-depleted mice following the above circulation studies and were stained with anti-ASPGR1/2 (liver; red) (b,c) or anti-F4/80 (spleen; red) (d). Nuclei were counterstained with 4',6-diamidino-2-phenylindole (blue). Arrows indicate co-localization of CFMDA-labelled platelets with ASPGR on hepatocytes. (e) Fluorescent platelet (green) localization was assessed with immunofluorescent microscopy at $\times 60$ magnification and quantified with Image J. White scale bars, $10 \mu \mathrm{M}$. ${ }^{\star} P<0.05$ as assessed by the Student's $t$-test. Data are representative of five randomly selected fluorescent images.

activation and desialylation, suggesting that anti-GPIb $\alpha$-mediated platelet effects predominantly follow $\mathrm{F}(\mathrm{ab})_{2}$ binding and are $\mathrm{Fc}$ independent. However, Fc $\gamma$ RIIa may exacerbate the anti-platelet effects and explain the stronger anti-GPIb $\alpha$-mediated responses seen in human versus mouse platelets. Through its tyrosine-based activation motif, Fc $\gamma$ RIIa signalling has been previously shown to contribute to, and was correlated with antibody-mediated platelet activation $^{31,50,51}$, particularly when associated with the GPIb complex $^{31,51,52}$. This may also be a target mechanism for Fc $\gamma \mathrm{R}$ dependent therapies, such as IVIG, in both anti-GPIIbIIIa and anti-GPIb $\alpha$ ITP patients who are responsive to the therapy ${ }^{53}$.

We thus show that the anti-platelet effects of anti-GPIb $\alpha$ are fundamentally different from those of anti-GPIIbIIIa, whereby anti-GPIb $\alpha$ antibodies in ITP crosslink adjacent GPIb $\alpha$ subunits causing GPIb $\alpha$-receptor clustering and activation. While we found that desialylation is dependent on anti-GPIb $\alpha$-induced platelet activation, we observed through sialidase inhibition assays that desialylation itself contributes to platelet activation. We propose that NEU1-mediated desialylation, through the removal of bulky sialic residues, facilitates further GPIb $\alpha$ clustering. As has been previously reported, GPIb complex clustering can induce platelet activation independent of GPIb agonist stimulation $^{41,44}$. It is also conceivable that removal of bulky sialic residues may facilitate anti-GPIb $\alpha$-antibody-mediated GPIb $\alpha$ crosslinking and signalling. These events synergize GPIb $\alpha$ signalling and platelet activation as demonstrated by increased P-selectin and NEU1 expressions. This indicates a positive feedback loop whereby platelet activation and NEU1-mediated 

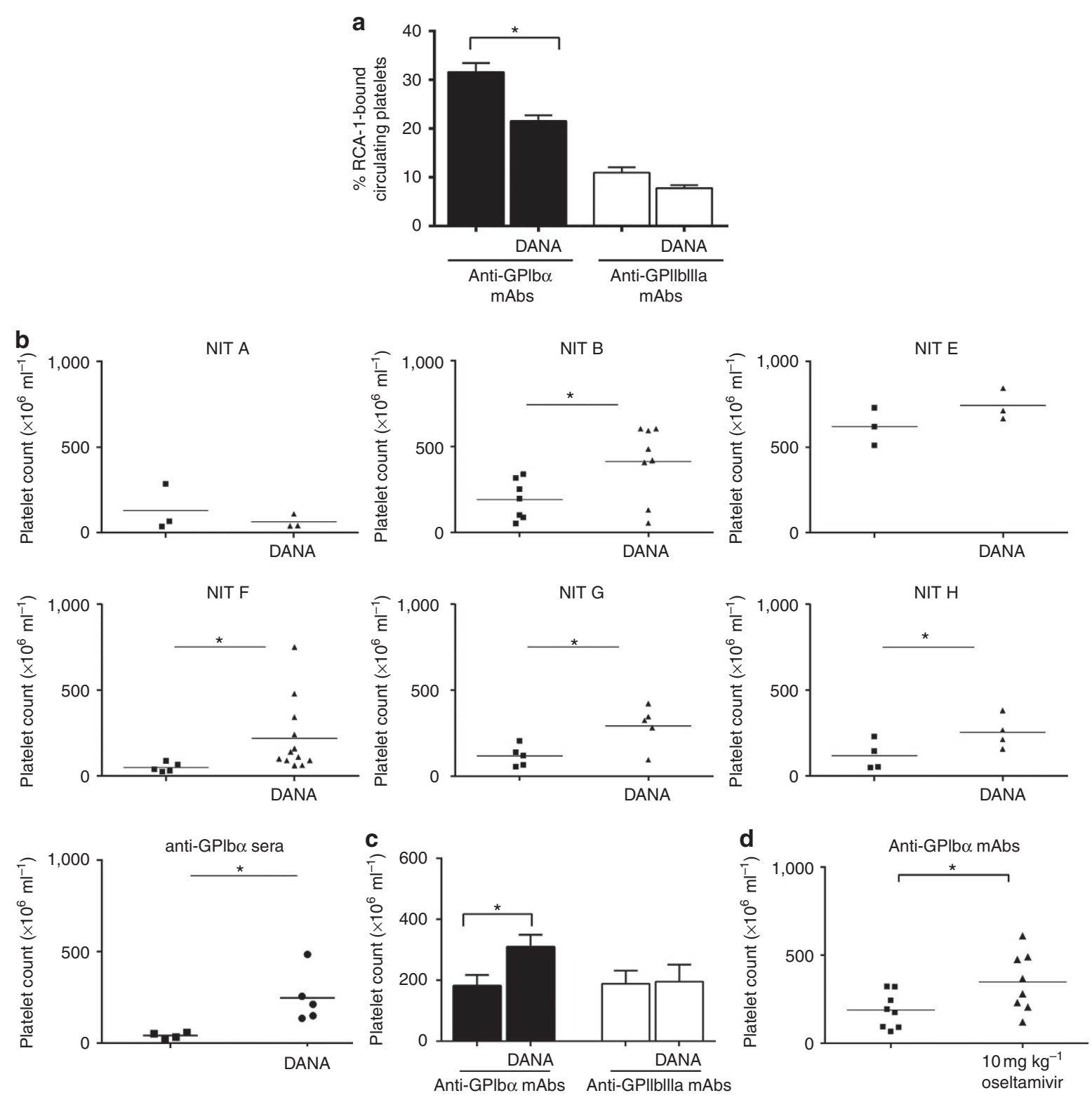

Figure 8 | Sialidase inhibition rescues thrombocytopenia in a murine model of ITP. (a-c) DANA or PBS was injected intraperitoneally immediately prior to anti-GPIb $\alpha$ or anti-GPIIbllla antibody injection (mAb or sera) to induce thrombocyotopenia. (a) Platelets from antibody-injected mice were analysed for desialylation (RCA-1 binding) via flow cytometry. (b) Platelet numbers were enumerated and compared between DANA-treated or mock (PBS)-treated groups. (c) Cumulative platelet counts from all anti-GPIb $\alpha$ and anti-GPIIbllla antibody (9D2, M1, PSI C1 and antisera)-injected mice with or without DANA treatment. For individual platelet counts of anti-GPIIbllla-injected mice, please see Supplementary Fig. 7. (d) Oseltamivir phosphate was injected intravenously $2 \mathrm{~h}$ after anti-GPIb $\alpha \mathrm{mAbs}$ injection (equal ratio mixture of individual $\mathrm{mAb}$ clones). Platelet enumeration was as described above. Data are shown as mean \pm s.e.m. platelet counts of individual mAbs. ${ }^{\star} P<0.05,{ }^{\star \star \star} P<0.001$ as determined by the Student's $t$-test.

desialylation reinforce each other, synergistically contributing to platelet clearance. This positive feedback loop may explain why relatively low levels of autoantibodies in ITP patients can cause severe thrombocytopenia.

To date, the prevailing view has been that platelet clearance in ITP is mediated by Fc $\gamma$ Rs of reticuloendothelial system macrophages, primarily in the spleen ${ }^{2}$. Here, we provide the first evidence of a new phagocytic process involving the hepatocyte in mediating destruction of antibody-opsonized platelets. Platelet clearance via the AMR is dominant in the absence of macrophages, which may be analogous to ITP patients undergoing typical first-line treatments. As hepatic sequestration of platelets in ITP patients is correlated with refractoriness to splenectomy ${ }^{54,55}$, our findings that the hepatocyte AMR plays a significant role in the clearance of anti-GPIb $\alpha$-opsonized desialylated platelets provide a potential explanation for refractoriness to splenectomy, as well as to steroid and IVIG therapies.

The burden on hepatocytes in the clearance of antibody-bound desialylated platelets has never been studied. However, increased production of liver stress enzymes has been reported in untreated ITP patients ${ }^{56}$. Given that the liver is an important site for production of plasma coagulation factors required for hemostasis $^{57}$, patients with anti-GPIb $\alpha$ antibodies may present with additional complications in the coagulation cascade and may experience a more severe bleeding phenotype. The liver is also the primary site for thrombopoietin production, thus there may be an indirect anti-GPIb $\alpha$ antibody effect on platelet production ${ }^{46}$. 
Long-term increased hepatic clearance of platelets may affect the coagulation system or decrease platelet production in anti-GPIb $\alpha$-mediated ITP, and warrants further study.

We show in $F c \gamma R^{-1-}$ and Aspgr $2^{-1-}$ mice that anti-GPIb $\alpha$ antibody-driven desialylation of platelet GPs significantly contributes to $\mathrm{Fc}$-independent thrombocytopenia in vivo. This may explain findings in a recent report of a fatal case of refractory ITP where incubation of the patients' sera (positive for anti-GPIb $\alpha$ antibodies only) with normal healthy human platelets caused significant platelet activation and desialylation ${ }^{58}$.

We found anti-GPIb $\alpha$-mediated platelet effects were susceptible to therapeutic intervention in vivo, as the sialidase inhibitors, DANA and oseltamivir, were able to rescue antiGPIb $\alpha$-mediated thrombocytopenia in mice. Clinical relevance of this finding is supported by a recent study in a small cohort of patients, which found a greater platelet increase in oseltamiviradministered individuals independent of influenza infection ${ }^{59}$. This follows a case report of the successful treatment of an anti-GPIb $\alpha$-positive ITP patient with oseltamivir, who had been previously refractory to numerous first- and second-line therapies. Remarkably, on oseltamivir, he maintained a sustained platelet response $e^{60}$. It is possible that the therapeutic targets of oseltamivir in these patients may not only be limited to platelets, as sialidase inhibition may also target immune cells, contributing to the amelioration of thrombocytopenia through modulation of the immune response ${ }^{61,62}$. Conversely, NEU1 released from platelets may adversely target the same immune cells and amplify the anti-platelet response ${ }^{63}$. In addition, non-platelet-derived sialidases causing enhanced removal of desialylated platelets, as has been reported in various infectious diseases such as Streptococcus pneumonia ${ }^{64}$ and Trypansoma cruzi (Chagas' disease) ${ }^{65}$, may provide links with infections and thrombocytopenia. This may also occur with influenza infection $^{66}$, although direct evidence is lacking to date.

These findings also provide insights into the platelet activation-desialylation dialogue and its positive feedback loop, which may contribute to thrombosis and cardiovascular diseases. Platelet activation and desialylation have been previously described in coronary heart disease (CHD) patients ${ }^{67,68}$, in which the sialic content of platelets was inversely correlated with the severity of $\mathrm{CHD}^{67}$. In addition, compared with patients without $\mathrm{CHD}$, CHD patients overall exhibited more desialylated platelets that were prone to aggregation with higher sensitivity to weak agonists, such as ADP and serotonin ${ }^{68,69}$. These reports strongly reinforce the notion that platelet desialylation potentiates activation of platelets in other disease states, which may result in aggregation and platelet consumption. Thus, this novel Fc-independent platelet clearance pathway may also be involved in the clearance of activated platelets or platelet microaggregates including embolized thrombi, and is likely relevant to other thrombocytopenias caused by antibodies, such as fetal/neonatal alloimmune thrombocytopenia, post-transfusion purpura and drug-induced thrombocytopenias.

In summary, we have demonstrated that anti-GPIb $\alpha$ antibodies cause platelet activation and desialylation, two processes coexisting in a positive feedback loop. This leads to Fc-independent platelet clearance in the liver mediated by the AMR of the hepatocyte. Most importantly, this pathway is amenable to sialidase inhibition. Given that IVIG is extremely costly and a limited resource, and steroids are associated with severe side effects, these treatments should only be implemented to patients who will benefit. Patients with anti-GPIb $\alpha$-mediated ITP who present with significant platelet desialylation may be identified as likely non-responders to conventional first-line treatments and splenectomy. We should also not exclude the potential impact of sialidase inhibitor therapy on anti-GPIIbIIIa-mediated ITP, as some anti-GPIIbIIIa antibodies may also cause platelet desialylation in human platelets. Clinical trials are required to test the diagnostic value of platelet desialylation and the therapeutic potential of sialidase inhibitors. Thus, our findings not only offer valuable implications for the diagnosis, prognosis and successful treatment of ITP, but also provide a potential mechanism in the clearance of activated platelets (emboli). As such, these findings may also have important implications in the field of alloimmune thrombocytopenias and cardiovascular diseases, although further investigation is required to test these hypotheses.

\section{Methods}

Mice. GPIb $\alpha^{-/-}$mice and GPIIIa $a^{-/-}$mice were originally described ${ }^{25}$ and provided by Dr Zaverio M. Ruggeri and Dr Jerry Ware (The Scripps Research Institute) and Dr Richard O. Hynes (Massachusetts Institute of Technology), respectively. The mice were backcrossed to BALB/c (Charles River Laboratories) background 10 times, then bred to generate syngeneic gene-deficient mice. $F c \gamma R^{-/-}$(C.129P2(B6)-Fcerlg ${ }^{\text {tm1Rav }}$ N12) (6-8 weeks) mice were purchased from Taconic. All aforementioned mice were housed in the St Michael's Hospital Research Vivarium, and all studies were approved by the St Michael's Hospital Animal Care Committee. Female mice between ages of 8 and 12 weeks were used for experiments. Aspgr $2^{-I-}$ mice and related experiments were performed by Dr. Karin Hoffmeister's laboratory at the Harvard Medical School.

Monoclonal antibody generation. GPIIIa ${ }^{-/-}$or $\mathrm{GPIba}^{-/-} \mathrm{BALB} / \mathrm{c}$ female mice were transfused with $10^{8}$ WT washed platelets per week, for 4-6 weeks. Immunized splenocytes were then fused with mouse myeloma cells Ag8.653, and hybridomas were selected by hypoxanthine-aminopterin-thymidine medium. Positive hybridomas were identified using flow cytometry and subcloned by limiting dilution. Antibody-secreting hybridomas were cultured in sera-free medium for large-scale antibody production. Monoclonal antibodies were purified using protein-G sepharose beads.

Platelet aggregation assay. Platelet aggregation was performed as previously described $^{28,26,27,70}$. Platelet-rich plasma (PRP) was prepared from citrated whole blood $(1: 9 \mathrm{v} / \mathrm{v})$ by centrifugation at $300 \mathrm{~g}$ for $7 \mathrm{~min}$. Gel-filtered platelets were purified from PRP using a Sepharose 2B chromatography column with PIPES buffer ( $5 \mathrm{mM}$ PIPES, $137 \mathrm{mM} \mathrm{NaCl}, 4 \mathrm{mM} \mathrm{KCl}$ and $0.1 \%$ glucose, $\mathrm{pH} 7.0$ ). Gel-filtered platelets or PRP $\left(250 \mu \mathrm{l}, 2.5 \times 10^{8} \mathrm{ml}^{-1}\right)$ were incubated with PBS, control IgG or anti-GPIb $\alpha$ or anti-GPIIbIIIa mAbs for $10 \mathrm{~min}$. To characterize anti-GPIIbIIIa antibodies, platelet aggregation was induced by $20 \mu \mathrm{M}$ ADP (for PRP) or $1 \mathrm{U} \mathrm{ml}^{-1}$ thrombin (for gel-filtered platelets). As for anti-GPIb $\alpha$ antibodies, platelet aggregation in PRP was induced by $20 \mu \mathrm{g} \mathrm{ml}^{-1}$ botrocetin (for mouse) or $1.5 \mathrm{mg} \mathrm{ml}^{-1}$ ristocetin (for human) and monitored by a computerized Chrono-log aggregometer (Chrono-Log Corporation, Havertown, PA, USA).

Patients. The patient plasma samples utilized in the present manuscript were from our previous published study of 176 ITP patients conducted at the Anhui Medical University Hospital, Hefei, China ${ }^{16}$. Informed consent was obtained from all subjects and the study was approved by the Anhui Medical University research ethics committee. Plasma samples were characterized with MAIPA, and only those samples highly specific with either anti-GPIb $\alpha$ with undetectable anti-GPIIbIIIa or anti-GPIIbIIIa with undetectable anti-GPIb $\alpha$ were selected for studies in this manuscript. Twenty-four untreated ITP patients' plasma samples were included: 12 with anti-GPIb $\alpha$ (patients ITP-1 to ITP-12) and 12 with anti-GPIIbIIIa (patients ITP-a to ITP-l) autoantibodies.

Reagents and materials. Inhibitors were used for the following: intracellular $\mathrm{Ca}^{2}+$ increases: BAPTA-AM (EMD4 Biosciences), P38MAPK: SB203580 (Enzo Life Sciences), Src family protein tyrosine kinase: PP1 (Sigma), neuraminidase: $\mathrm{N}$-acetyl-2,3-dehydro-2-deoxy neuraminic acid sodium salt (DANA) (EMD4 Biosciences) and oseltamivir phosphate (Santa Cruz), pan-caspases: Q-VD-OPh (Sigma), cAMP: prostacyclin $\left(\mathrm{PGI}_{2}\right)$ (Cayman) and AMR: asialofetuin and its control fetuin (Sigma). OSGE (Cedarlane) was used to cleave GPIb $\alpha$.

Blood collection and platelet isolation. Procedures were approved by the Research Ethics Board of St Michael's Hospital (Toronto, ON, Canada) and conducted as previously described $25,26,58$. Venous blood was obtained from healthy volunteers by venipuncture into $3.2 \%$ trisodium citrate. PRP was prepared by centrifugation $\left(10 \mathrm{~min}, 300 \mathrm{~g}\right.$, no brake, $\left.22^{\circ} \mathrm{C}\right)$. Platelets were isolated from PRP and washed $\left(15 \mathrm{~min}, 1,050 \mathrm{~g}\right.$, no brake, $22^{\circ} \mathrm{C}$, with $\left.10 \mathrm{ng} \mathrm{ml}^{-1} \mathrm{PGI}_{2}\right)$ and resuspended in HEPES Tyrode's solution ( $145 \mathrm{mM} \mathrm{NaCl}, 5 \mathrm{mM} \mathrm{KCl}, 0.5 \mathrm{mM}$

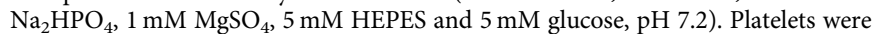
counted with a Z2 Series Coulter Counter (Beckman Coulter), adjusted to $200 \times 10^{6}$ platelets per $\mathrm{ml}$ and stored at $22^{\circ} \mathrm{C}$ for $30 \mathrm{~min}$ to regain responsiveness. 
Sera were isolated from blood of healthy donors by high-speed centrifugation of the platelet-poor plasma and red blood cell fraction $\left(5 \mathrm{~min}, 2,000 \mathrm{~g}, 22^{\circ} \mathrm{C}\right)$. For isolation of murine platelets, blood was collected from anesthetized mice in $100 \mu \mathrm{l}$ of $130 \mathrm{mM}$ trisodium citrate by retro-orbital eye bleed, and washed platelets were prepared as described above with human-washed platelets.

In vitro platelet-antibody assays. Platelets were treated with various $\mathrm{mAbs}$ $\left(2.5 \mu \mathrm{g} \mathrm{ml}^{-1}\right)$ or with sera from $\mathrm{GPIb}^{-/-}$or GPIIIa ${ }^{-/-}$mice that have been intravenously immunized $4 \times$ weekly with $10^{8}$ WT platelets ${ }^{25}$. Human platelets were also treated with sera from ITP patients $(1 / 50 \mathrm{v} / \mathrm{v})$ in HEPES Tyrode's solution, for $60 \mathrm{~min}$ at $22^{\circ} \mathrm{C}$. Unbound antibody was removed by centrifugation (15 min, $1,050 \mathrm{~g}$, no brake, $22^{\circ} \mathrm{C}$, with $10 \mathrm{ng} \mathrm{ml}^{-1} \mathrm{PGI}_{2}$ ). Platelets were resuspended in Tyrode's solution ( $\mathrm{pH} 7.2)$ and incubated $\left(30 \mathrm{~min}, 22^{\circ} \mathrm{C}\right)$ to restore responsiveness before measurements. For visualization of antibody-mediated platelet aggregate formation, human or murine PRP were incubated with various $\mathrm{mAb}\left(2.5 \mu \mathrm{g} \mathrm{ml}^{-1}\right)$ under stirring conditions (1,000 r.p.m.) for $10 \mathrm{~min}$. The images of platelet aggregates were recorded using a digital camera (DP70; Olympus, Tokyo, Japan) under a Zeiss Axiovert 135 inverted fluorescent microscope (Zeiss, Oberkochen, Germany). In some experiments, various inhibitors were used, prior to addition of $\mathrm{mAbs}\left(15 \mathrm{~min}, 22^{\circ} \mathrm{C}\right)$, P38MAPK inhibitor SB203580 $(10 \mu \mathrm{M})$, $\mathrm{Ca}^{2+}$ quencher BAPTA-AM $(20 \mu \mathrm{M})$, Src family protein tyrosine kinase inhibitor PP1 $(10 \mu \mathrm{M})$, Q-VD-OPh $(50 \mu \mathrm{M})$, neuraminidase inhibitor DANA $(1 \mathrm{mM})$ or Fc $\gamma$ RIIa/III inhibitor IV.3 (StemCell Technologies) $\left(2.5 \mu \mathrm{g} \mathrm{ml}^{-1}\right)$. In some experiments OSGE $\left(80 \mu \mathrm{g} \mathrm{ml}^{-1}\right)$ was used to remove the extracellular part of GPIb $\alpha$ following antibody incubation, as previously described ${ }^{43}$.

Flow cytometry. P-selectin expression was detected with FITC-labelled anti-Pselectin antibody $\left(5 \mu \mathrm{g} \mathrm{ml}^{-1}\right)$ (BD Biosciences) for murine platelets, or with PE-labelled $\left(2 \mu \mathrm{g} \mathrm{ml}^{-1}\right)$ (eBioscience) or Brilliant Violet $605\left(0.5 \mu \mathrm{g} \mathrm{ml}^{-1}\right)$ (Biolegend) anti-human CD62P (P-selectin) antibody for human platelets. GPIIbIIIa activation was detected with JON/A-PE (5 $\mu \mathrm{l}$ per $10^{6}$ platelets) (Emfret) or PAC-1-FITC (20 $\mu \mathrm{l}$ per test) (BD Biosciences) following platelet fixation in $1 \%$ paraformaldehyde for $15 \mathrm{~min}$ on murine and human platelets, respectively. To quantify glycosylation, platelets were incubated with fluorescein-labelled RCA-1 or fluorescein-labelled PNA or biotinylated Maackia Amurensis Lectin II or fluorescein-labelled Sambucus Nigra Lectin. All were purchased from Vector Laboratories and used at $0.5 \mu \mathrm{g} \mathrm{ml}-1,15 \mathrm{~min}, 22^{\circ} \mathrm{C}$ to measure exposure of sugar residues. Surface NEU1 expression was measured with rabbit anti-NEU1 antibody (clone H-300 Santa Cruz, 1:50 dilution) and detected with Alexa Fluor 647 secondary antibody $\left(0.8 \mu \mathrm{g} \mathrm{ml}^{-1}\right)$ (Molecular Probes/Invitrogen). Murine IgG binding was detected with Alexa Fluor 647 goat anti-mouse IgG $(\mathrm{H}+\mathrm{L})$ $\left(0.8 \mu \mathrm{g} \mathrm{ml}^{-1}\right)$ (Invitrogen) or. Fold increase is represented as percentage increase from gated control platelets set at $1 \%$ positive. A total of 10,000 platelet events were acquired and analysed on a MACSQuant (Miltenyi Biotech).

Western blotting. Human platelets were incubated with mAb (NIT A, NIT B or NIT F) and DANA or $10 \mu \mathrm{M}$ PP1, or $10 \mu \mathrm{M} \mathrm{SB} 253580$ as described above and lysed in $1 \times$ Laemmli buffer. For detection of phospho-p38MAPK, $1 \times$ Halt protease inhibitor cocktail (Thermo Scientific) was also added. Samples were reduced with addition of $0.1 \mathrm{M}$ dithiothreitol and boiled at $95^{\circ} \mathrm{C}$ for $10 \mathrm{~min}$. Platelet lysate was separated by SDS-polyacrylamide gel electrophoresis, transferred onto polyvinylidene difluoride membrane (GE Healthcare). For RCA-1 staining, membranes were probed with biotin-conjugated RCA-1 $\left(2 \mu \mathrm{g} \mathrm{ml}^{-1}\right)$ (Vector Labs) according to the manufacturer's instructions, mouse anti-human CD41b antibody $(1: 5,000)$ (clone EPR6995, Abcam) and with $\beta$-actin antibody (1:400) (clone $\mathrm{Cl}$, Santa Cruz). For phospho-p38MAPK, membranes were probed with phosphop38MAPK (Thr180/Tyr182) antibody (1:5,000) (Cell Signaling Techonology) at $4{ }^{\circ} \mathrm{C}$ overnight. Avidin conjugation to RCA-1 was performed with a Vectastain $\mathrm{ABC}$ kit (Vector Labs) according to the manufacturer's instructions. HRPconjugated secondary anti-mouse antibody $(1: 5,000)$ (Santa Cruz) was then utilized. Blots were developed by ECL (Thermo Scientific) and visualized on the VersaDoc MP 4000 imaging system (Bio-Rad). Uncropped immunoblots are shown in Supplementary Fig. 9.

Antibody fab generation. NIT A, NIT B and NIT F Fab fragments were generated with Thermo Scientific IgG1 $\mathrm{F}(\mathrm{Ab})_{2}$ and Fab preparation kits according to manufacturers' protocol. Briefly, $1 \mathrm{mg} \mathrm{ml}^{-1}$ of antibody was desalted and digested with immobilized Ficin (beaded agarose resin) in the presence of $25 \mathrm{mM}$ cysteine. Digest products were purified with Protein-G beads and dialysed and concentrated with Amicon Ultra-4 Centrifugal Filter Units (Millipore). Purified Fab was run on $10 \%$ SDS-polyacrylamide gel for visualization.

Immunocytochemistry. Platelets treated with anti-GPIb $\alpha$ or anti-GPIIbIIIa antibodies as described above were fixed in $4 \%$ paraformaldehyde at $22^{\circ} \mathrm{C}$ for $20 \mathrm{~min}$ and spun down onto poly-L-lysine-coated coverslips (BD Biosciences; $500 \mathrm{~g}, 5 \mathrm{~min}$ ). Cells were blocked overnight in PBS with $1 \%$ BSA at $4{ }^{\circ} \mathrm{C}$. Cells were incubated with primary antibodies rabbit anti-NEU1 $\operatorname{IgG}\left(4 \mu \mathrm{g} \mathrm{ml}^{-1}\right.$ ) (Santa Cruz) and our anti-CD61 mAb M1 $\left(5 \mu \mathrm{g} \mathrm{ml}^{-1}\right)$ overnight at $4^{\circ} \mathrm{C}$. The platelets were washed in triplicate with PBS before addition of species appropriate secondary antibodies Alexa Fluor 488 and $647(1: 10,000)$ (Invitrogen). Images were taken with a Zeiss LSM 700 Confocal laser scanning microscope with a $\times 63$ objective. Images were analysed with ZEN 2010 software (Zeiss) and Adobe Photoshop 7.

In vivo platelet activation and desialylation. The passive murine ITP model was used to induce thrombocytopenia ${ }^{12}$. Various anti-platelet $\mathrm{mAbs}$ were injected intraperitoneally. The amount of mAbs used was determined following titration to induce similar levels of thrombocytopenia. After titration, $0.75 \mu \mathrm{g}$ of anti-GPIb $\alpha$ $\mathrm{mAb}\left(0.03 \mu \mathrm{gg}^{-1}\right.$ total body weight) was found to be comparable to injection of $4 \mu \mathrm{g}$ of anti-GPIIbIIIa $\mathrm{mAb}\left(0.16 \mu \mathrm{gg}^{-1}\right.$ total body weight). In some experiments, neuraminidase inhibitor DANA ( $2 \mathrm{mg}$ or $0.08 \mathrm{mgg}^{-1}$ total body weight) was injected intraperitoneally $2 \mathrm{~min}$ prior to injection of mAbs. Alternatively, oseltamivir phosphate $\left(0.25 \mathrm{mg}\right.$ or $0.01 \mathrm{mgg} \mathrm{g}^{-1}$ total body weight) was therapeutically intravenously injected $2 \mathrm{~h}$ after antibody injection. After $16 \mathrm{~h}$, mice were bled via the saphenous vein, platelet count was measured with Coulter $\mathrm{Z}$ counter and PRP was analysed for P-selectin and RCA-1 binding via flow cytometry as described above.

In vivo platelet clearance. A total of $10^{8}$ washed murine platelets were prepared as described above and labelled with $5 \mu \mathrm{M}$ of CMFDA and incubated with antiplatelet mAbs $\left(5 \mu \mathrm{g} \mathrm{ml}^{-1}, 1 \mathrm{~h}, 22^{\circ} \mathrm{C}\right)$, washed and injected intravenously into WT BALB/c mice. Platelet circulation was measured after transfusion of CMFDAlabelled platelets via enumeration of fluorescently labelled platelets in whole-blood samples after 1 (baseline), 15 and $30 \mathrm{~min}$. To assess the contribution of AMRs on hepatocytes, a bolus injection of inhibitor asialofetuin $\left(0.2 \mathrm{mg} \mathrm{g}^{-1}\right.$ total body weight) was administered via the tail vein and fetuin was used as control, as previously described ${ }^{24}$. At $10 \mathrm{~min}$ post transfusion, a booster injection of asialofetuin $\left(0.1 \mathrm{mgg}^{-1}\right.$ total body weight) was administered intraperitoneally. Some mice were depleted of their macrophages via intravenous injection of Clondrate-encapsulated liposomes $\left(0.01 \mathrm{mlg}^{-1}\right.$ body weight $) 48 \mathrm{~h}$ prior.

Immunohistochemistry. Following in vivo platelet clearance studies as described above, mice were anesthetized and drained of their blood via retro-orbital bleeding. Liver and spleen were harvested and snap-frozen in liquid nitrogen. Frozen tissue was sectioned $(5 \mu \mathrm{m})$ with Leica Cryostat and fixed onto slides in ice-cold methanol. Slides were washed in $2 \% \mathrm{BSA}$ and then incubated with primary rat anti-mouse F4/80 (1:1,000) (clone BM8, eBioscience) or rabbit anti-ASPGR1/1 $(1: 1,000)$ (Santa Cruz) overnight at $4{ }^{\circ} \mathrm{C}$. Slides were then stained or anti-rat Cy3 or anti-rabbit Alexa Fluor 647 secondary antibody $(1: 5,000)$ for $2 \mathrm{~h}$. Sections were mounted with Vectashield mounting medium containing 4',6-diamidino-2phenylindole. Images were captured using Olympus upright fluorescence microscope and analysed with Adobe Photoshop 7 and Image J.

Statistical analysis. All data are presented as mean \pm s.e.m. unless otherwise indicated. Statistically significant differences between controls were assessed by the Student's unpaired $t$-test (two tailed). Statistically significant differences between multiple groups were assessed by one-way or two-way analysis of variance with Bonferroni post hoc analysis, as indicated. Statistical analyses were performed using Prism software (GraphPad). A $P$ value of 0.05 or less was considered significant.

\section{References}

1. Rodeghiero, F. et al. Standardization of terminology, definitions and outcome criteria in immune thrombocytopenic purpura of adults and children: report from an international working group. Blood 113, 2386-2393 (2009).

2. McMillan, R. The pathogenesis of chronic immune thrombocytopenic purpura. Semin. Hematol. 44, S3-S11 (2007).

3. Cines, D. B., Bussel, J. B., Liebman, H. A. \& Luning Prak, E. T. The ITP syndrome: pathogenic and clinical diversity. Blood 113, 6511-6521 (2009).

4. Mathias, S. D. et al. Impact of chronic Immune Thrombocytopenic Purpura (ITP) on health-related quality of life: a conceptual model starting with the patient perspective. Health Qual. Life Outcomes 6, 13 (2008).

5. Provan, D. et al. International consensus report on the investigation and management of primary immune thrombocytopenia. Blood 115, 168-186 (2010).

6. Vianelli, N. et al. Efficacy and safety of splenectomy in immune thrombocytopenic purpura: long-term results of 402 cases. Haematologica $\mathbf{9 0}$, 72-77 (2005)

7. Neunert, C. et al. The American Society of Hematology 2011 evidence-based practice guideline for immune thrombocytopenia. Blood 117, 4190-4207 (2011).

8. Harrington, W. J., Minnich, V., Hollingsworth, J. W. \& Moore, C. V. Demonstration of a thrombocytopenic factor in the blood of patients with thrombocytopenic purpura. J. Lab. Clin. Med. 38, 1-10 (1951).

9. Beardsley, D. S. \& Ertem, M. Platelet autoantibodies in immune thrombocytopenic purpura. Transfus. Sci. 19, 237-244 (1998). 
10. Bussel, J. B. Fc receptor blockade and immune thrombocytopenic purpura. Semin. Hematol. 37, 261-266 (2000).

11. Nieswandt, B., Bergmeier, W., Rackebrandt, K., Gessner, J. E. \& Zirngibl, H. Identification of critical antigen-specific mechanisms in the development of immune thrombocytopenic purpura in mice. Blood 96, 2520-2527 (2000).

12. Webster, M. L. et al. Relative efficacy of intravenous immunoglobulin G in ameliorating thrombocytopenia induced by antiplatelet GPIIbIIIa versus GPIbalpha antibodies. Blood 108, 943-946 (2006).

13. Go, R. S., Johnston, K. L. \& Bruden, K. C. The association between platelet autoantibody specificity and response to intravenous immunoglobulin $\mathrm{G}$ in the treatment of patients with immune thrombocytopenia. Haematologica 92, 283-284 (2007).

14. Peng, J. et al. Association of autoantibody specificity and response to intravenous immunoglobulin $\mathrm{G}$ therapy in immune thrombocytopenia: a multicenter cohort study. J. Thromb. Haemost. 12, 497-504 (2014).

15. Chang, M. et al. Immune thrombocytopenic purpura (ITP) plasma and purified ITP monoclonal autoantibodies inhibit megakaryocytopoiesis in vitro. Blood 102, 887-895 (2003).

16. Zeng, Q. et al. Relative efficacy of steroid therapy in immune thrombocytopenia mediated by anti-platelet GPIIbIIIa versus GPIbalpha antibodies. Am. J. Hematol. 87, 206-208 (2012).

17. Ozaki, Y., Asazuma, N., Suzuki-Inoue, K. \& Berndt, M. C. Platelet GPIb-IX-V-dependent signaling. J. Thromb. Haemost. 3, 1745-1751 (2005).

18. Shattil, S. J. \& Newman, P. J. Integrins: dynamic scaffolds for adhesion and signaling in platelets. Blood 104, 1606-1615 (2004).

19. Du, X. Signaling and regulation of the platelet glycoprotein Ib-IX-V complex. Curr. Opin. Hematol. 14, 262-269 (2007).

20. Okumura, I., Lombart, C. \& Jamieson, G. A. Platelet glycocalicin. II. Purification and characterization. J. Biol. Chem. 251, 5950-5955 (1976).

21. Solum, N. O., Hagen, I., Filion-Myklebust, C. \& Stabaek, T. Platelet glycocalicin. Its membrane association and solubilization in aqueous media. Biochim. Biophys. Acta 597, 235-246 (1980).

22. Rumjantseva, V. et al. Dual roles for hepatic lectin receptors in the clearance of chilled platelets. Nat. Med. 15, 1273-1280 (2009).

23. Wandall, H. H. et al. Galactosylation does not prevent the rapid clearance of long-term, 4 degrees C-stored platelets. Blood 111, 3249-3256 (2008).

24. Sorensen, A. L. et al. Role of sialic acid for platelet life span: exposure of beta-galactose results in the rapid clearance of platelets from the circulation by asialoglycoprotein receptor-expressing liver macrophages and hepatocytes. Blood 114, 1645-1654 (2009).

25. Li, C. et al. The maternal immune response to fetal platelet GPIbalpha causes frequent miscarriage in mice that can be prevented by intravenous IgG and anti-FcRn therapies. J. Clin. Invest. 121, 4537-4547 (2011).

26. Lei, X. et al. Anfibatide, a novel GPIb complex antagonist, inhibits platelet adhesion and thrombus formation in vitro and in vivo in murine models of thrombosis. Thromb. Haemost. 111, 279-289 (2014).

27. Yang, H. et al. Fibrinogen and von Willebrand factor-independent platelet aggregation in vitro and in vivo. J. Thromb. Haemost. 4, 2230-2237 (2006).

28. Reheman, A. et al. Plasma fibronectin depletion enhances platelet aggregation and thrombus formation in mice lacking fibrinogen and von Willebrand factor. Blood 113, 1809-1817 (2009).

29. Wang, Y. et al. Plasma fibronectin supports hemostasis and regulates thrombosis. J. Clin. Invest. 124, 4281-4293 (2014).

30. Yanabu, M. et al. Tyrosine phosphorylation and p72syk activation by an anti-glycoprotein Ib monoclonal antibody. Blood 89, 1590-1598 (1997).

31. Cauwenberghs, N. et al. Fc-receptor dependent platelet aggregation induced by monoclonal antibodies against platelet glycoprotein $\mathrm{Ib}$ or von Willebrand factor. Thromb. Haemost. 85, 679-685 (2001).

32. Shattil, S. J., Hoxie, J. A., Cunningham, M. \& Brass, L. F. Changes in the platelet membrane glycoprotein IIb.IIIa complex during platelet activation. J. Biol. Chem. 260, 11107-11114 (1985).

33. Bergmeier, W. et al. Flow cytometric detection of activated mouse integrin alphaIIbbeta3 with a novel monoclonal antibody. Cytometry 48, 80-86 (2002).

34. King, M., McDermott, P. \& Schreiber, A. D. Characterization of the Fc gamma receptor on human platelets. Cell. Immunol. 128, 462-479 (1990).

35. Pfueller, S. L. \& Luscher, E. F. The effects of aggregated immunoglobulins on human blood platelets in relation to their complement-fixing abilities. II. Structural requirements of the immunoglobulin. J. Immunol. 109, 526-533 (1972).

36. Hoffmeister, K. M. et al. Glycosylation restores survival of chilled blood platelets. Science 301, 1531-1534 (2003).

37. Zhang, Y. et al. Identification of selective inhibitors for human neuraminidase isoenzymes using C4,C7-modified 2-deoxy-2,3-didehydro-N-acetylneuraminic acid (DANA) analogues. J. Med. Chem. 56, 2948-2958 (2013).

38. Judson, P. A., Anstee, D. J. \& Clamp, J. R. Isolation and characterization of the major oligosaccharide of human platelet membrane glycoprotein GPIb. Biochem. J. 205, 81-90 (1982).
39. Kinlough-Rathbone, R. L., Perry, D. W., Rand, M. L. \& Packham, M. A. Responses to aggregating agents after cleavage of GPIb of human platelets by the O-sialoglycoprotein endoprotease from Pasteurella haemolytica- potential surrogates for Bernard-Soulier platelets? Thromb. Res. 99, 165-172 (2000).

40. Jansen, A. J. et al. Desialylation accelerates platelet clearance after refrigeration and initiates GPIbalpha metalloproteinase-mediated cleavage in mice. Blood 119, 1263-1273 (2012).

41. van der Wal, D. E. et al. Role of glycoprotein Ibalpha mobility in platelet function. Thromb. Haemost. 103, 1033-1043 (2010).

42. Gitz, E. et al. Improved platelet survival after cold storage by prevention of glycoprotein Ibalpha clustering in lipid rafts. Haematologica 97, 1873-1881 (2012).

43. van der Wal, D. E. et al. Platelet apoptosis by cold-induced glycoprotein Ibalpha clustering. J. Thromb. Haemost. 8, 2554-2562 (2010).

44. Kasirer-Friede, A. et al. Lateral clustering of platelet GP Ib-IX complexes leads to up-regulation of the adhesive function of integrin alpha IIbbeta 3. J. Biol. Chem. 277, 11949-11956 (2002).

45. Marshall, J. S. et al. Measurement of circulating desialylated glycoproteins and correlation with hepatocellular damage. J. Clin. Invest. 54, 555-562 (1974).

46. Grozovsky, R. et al. The Ashwell-Morell receptor regulates hepatic thrombopoietin production via JAK2-STAT3 signaling. Nat. Med. 21, 47-54 (2015).

47. Alimardani, G., Guichard, J., Fichelson, S. \& Cramer, E. M. Pathogenic effects of anti-glycoprotein Ib antibodies on megakaryocytes and platelets. Thromb. Haemost. 88, 1039-1046 (2002).

48. Cadroy, Y. et al. Relative antithrombotic effects of monoclonal antibodies targeting different platelet glycoprotein-adhesive molecule interactions in nonhuman primates. Blood 83, 3218-3224 (1994).

49. Cauwenberghs, N. et al. Antithrombotic effect of platelet glycoprotein Ib-blocking monoclonal antibody Fab fragments in nonhuman primates. Arterioscler. Thromb. Vasc. Biol. 20, 1347-1353 (2000).

50. Rubinstein, E., Kouns, W. C., Jennings, L. K., Boucheix, C. \& Carroll, R. C. Interaction of two GPIIb/IIIa monoclonal antibodies with platelet Fc receptor (Fc gamma RII). Br. J. Haematol. 78, 80-86 (1991).

51. Sullam, P. M. et al. Physical proximity and functional interplay of the glycoprotein Ib-IX-V complex and the Fc receptor FcgammaRIIA on the platelet plasma membrane. J. Biol. Chem. 273, 5331-5336 (1998).

52. Shrimpton, C. N. et al. Localization of the adhesion receptor glycoprotein Ib-IX-V complex to lipid rafts is required for platelet adhesion and activation. J. Exp. Med. 196, 1057-1066 (2002).

53. Urbanus, R. T. et al. Patient autoantibodies induce platelet destruction signals via raft-associated glycoprotein Ibalpha and FcgammaRIIa in immune thrombocytopenia. Haematologica 98, e70-e72 (2013).

54. Sarpatwari, A. et al. Autologous 111 In-labelled platelet sequestration studies in patients with primary immune thrombocytopenia (ITP) prior to splenectomy: a report from the United Kingdom ITP Registry. Br. J. Haematol. 151, 477-487 (2010).

55. Ghanima, W., Godeau, B., Cines, D. B. \& Bussel, J. B. How I treat immune thrombocytopenia: the choice between splenectomy or a medical therapy as a second-line treatment. Blood 120, 960-969 (2012).

56. Enger, C. et al. Hepatobiliary laboratory abnormalities among patients with chronic or persistent immune thrombocytopenia (ITP). Ann. Hepatol. 10, 188-195 (2011).

57. Davidson, C. J., Tuddenham, E. G. \& McVey, J. H. 450 million years of hemostasis. J. Thromb. Haemost. 1, 1487-1494 (2003).

58. Li, J. et al. Severe platelet desialylation in a patient with glycoprotein Ib/IX antibody-mediated immune thrombocytopenia and fatal pulmonary hemorrhage. Haematologica 99, e61-e63 (2014).

59. Jansen, A. J., Peng, J., Zhao, H. G., Hou, M. \& Ni, H. Sialidase inhibition to increase platelet counts: a new treatment option for thrombocytopenia. Am. J. Hematol. 90, E94-E95 (2015).

60. Shao, L. et al. Successful treatment with oseltamivir phosphate in a patient with chronic immune thrombocytopenia positive for anti-GPIb/IX autoantibody. Platelets 26, 495-497 (2014).

61. Nan, X., Carubelli, I. \& Stamatos, N. M. Sialidase expression in activated human T lymphocytes influences production of IFN-gamma. J. Leukoc. Biol. 81, 284-296 (2007).

62. Chen, X. P., Enioutina, E. Y. \& Daynes, R. A. The control of IL-4 gene expression in activated murine $\mathrm{T}$ lymphocytes: a novel role for neu-1 sialidase. J. Immunol. 158, 3070-3080 (1997).

63. Seyrantepe, V. et al. Regulation of phagocytosis in macrophages by neuraminidase 1. J. Biol. Chem. 285, 206-215 (2010).

64. Grewal, P. K. et al. The Ashwell receptor mitigates the lethal coagulopathy of sepsis. Nature medicine 14, 648-655 (2008).

65. Tribulatti, M. V., Mucci, J., Van Rooijen, N., Leguizamon, M. S. \& Campetella, $\mathrm{O}$. The trans-sialidase from Trypanosoma cruzi induces thrombocytopenia during acute Chagas' disease by reducing the platelet sialic acid contents. Infect. Immun. 73, 201-207 (2005). 
66. Alioglu, B., Tasar, A., Ozen, C., Selver, B. \& Dallar, Y. An experience of oseltamivir phosphate (tamiflu) in a pediatric patient with chronic idiopathic thrombocytopenic purpura: a case report. Pathophysiol. Haemost. Thromb. 37, 55-58 (2010).

67. Mandic, R., Opper, C., Krappe, J. \& Wesemann, W. Platelet sialic acid as a potential pathogenic factor in coronary heart disease. Thromb. Res. 106, 137-141 (2002).

68. Butun, I. I. et al. Preliminary study showing the relationship between platelet fibronectin, sialic acid, and ADP-induced aggregation levels in coronary heart disease. Clin. Appl. Thromb. Hemost. 13, 308-312 (2007).

69. McGill, D. A. \& Ardlie, N. G. Abnormal platelet reactivity in men with premature coronary heart disease. Coron. Artery Dis. 5, 889-900 (1994).

70. Wang, Y. et al. Tyrosine phosphatase MEG2 modulates murine developmen and platelet and lymphocyte activation through secretory vesicle function. J. Exp. Med. 202, 1587-1597 (2005).

\section{Acknowledgements}

We are grateful to Dr Z. Ruggeri and Dr Jerry Ware, and Dr R. Hynes for providing the GPIb $\alpha^{-/-}$and GPIIIa ${ }^{-/-}$mice, respectively. Alexandra H. Marshall provided valuable comments and contributed significantly to manuscript preparation. This work was supported by the Canadian Institutes of Health Research (MOP 97918, MOP 119540 and MOP 119551); Canadian Blood Services-Canadian Institutes of Health Research partnership fund (CIHR-BUC201403-HN-326400); Equipment Funds from the Canada Foundation for Innovation (Leaders Opportunity Fund: 31101), Canadian Blood Services and St Michael's Hospital. D.E.v.d.W., I.Y. and L.M. are recipients of the Canadian Blood Services Postdoctoral Fellowship. J.L. and B.V. are recipients of the Fellowship award from the Department of Laboratory Medicine and Pathobiology, University of Toronto.

\section{Author contributions}

J.L. designed and performed the majority of experiments, analysed data and prepared the manuscript. D.E.v.d.W. designed and conducted experiments and analyzed data. G.Z. created the mAbs and provided guidance for study design and data interpretation. M.X., R.G., I.Y. and B.V. conducted some of the experiments. L.M. provided polyclonal sera. M.R., L.Z. and Q.Z. led the clinical study in Hefei and provided blood samples to the group in Toronto, Canada. V.L., K.M.H. and J.F. contributed to study design and interpretation, as well as manuscript preparation. As principle investigator, H.N. oversaw study design, data interpretation and manuscript preparation.

\section{Additional information}

Supplementary Information accompanies this paper at http://www.nature.com/ naturecommunications

Competing financial interests: The authors declare no competing financial interests.

Reprints and permission information is available online at http://npg.nature.com/ reprintsandpermissions/

How to cite this article: Li, J. et al. Desialylation is a mechanism of Fc-independent platelet clearance and a therapeutic target in immune thrombocytopenia. Nat. Commun. 6:7737 doi: 10.1038/ncomms8737 (2015).

\section{(c) (i)}

This work is licensed under a Creative Commons Attribution 4.0 International License. The images or other third party material in this article are included in the article's Creative Commons license, unless indicated otherwise in the credit line; if the material is not included under the Creative Commons license, users will need to obtain permission from the license holder to reproduce the material To view a copy of this license, visit http://creativecommons.org/licenses/by/4.0/ 